Check for updates

Cite this: RSC Adv., 2019, 9, 13749

Received 28th February 2019

Accepted 17th April 2019

DOI: 10.1039/c9ra01529a

rsc.li/rsc-advances

\section{Lanthanide complexes combined with chiral salen ligands: application in the enantioselective epoxidation reaction of $\alpha, \beta$-unsaturated ketones $\uparrow$}

\author{
Xuexiu Xia, ${ }^{a}$ Chengrong Lu, ${ }^{* a}$ Bei Zhao iD *ab and Yingming Yao (D) ab
}

Readily available lanthanide amides $\operatorname{Ln}\left[\mathrm{N}\left(\mathrm{SiMe}_{3}\right)_{2}\right]_{3}(\mathrm{Ln}=\mathrm{Nd}(1), \mathrm{Sm}$ (2), $\mathrm{Eu}(3), \mathrm{Yb}(4), \mathrm{La}(5))$, combined with chiral salen ligands $\mathrm{H}_{2} \mathrm{~L}^{\mathrm{a}}\left((\mathrm{S}, \mathrm{S})-\mathrm{N}, \mathrm{N}^{\prime}\right.$-di-(3,5-disubstituted-salicylidene)-1,2-cyclohexanediamine) and $\mathrm{H}_{2} \mathrm{~L}^{\mathrm{b}}$ $\left((S, S)-N, N^{\prime}\right.$-di-(3,5-disubstituted-salicylidene)-1,2-diphenyl-1,2-ethanediamine) were employed in the enantioselective epoxidation of $\alpha, \beta$-unsaturated ketones. It was found that the salen-La complex shows the highest efficiency and enantioselectivity. A relatively broad scope of $\alpha, \beta$-unsaturated ketones was investigated, and excellent yields (up to 99\%) and moderate to good enantioselectivities (37-87\%) of the target molecules were achieved.

\section{Introduction}

Salen ligands are a particular class of Schiff bases, which are produced by condensation of two salicylaldehydes with a diamine. The use of salen metal complexes for a large variety of catalytic transformations has emerged, mainly because the salen ligands are easily available and highly tunable, and are capable of coordinating to many different metals to form various catalysts. ${ }^{1}$ In recent years, chiral salen ligands have become one of the most popular chiral ligands and a series of salen-transition metal catalysts have found application in enantioselective synthesis. In 2001, Kozlowski developed a set of modular bifunctional salen ligands, which showed enhanced reactivity in the asymmetric addition of diethylzinc to aldehydes and achieved excellent yield (up to 99\%) with high enantioselectivity (up to $91 \%$ ). ${ }^{2}$ In 2003 , Jiang used salen-Ti complexes to catalyze the enantioselective addition of TMSCN to ketones. ${ }^{3}$ Rawal reported that the enantioselective carbonyl-ene reactions were catalyzed by a salen-Co complex, which generated chiral homoallylic alcohols in excellent yields, enantioselectivities and diastereoselectivities. ${ }^{4}$ In 2014 , the asymmetric conia-ene-type cyclization of $\alpha$-functionalized ketones was catalyzed by a chiral salen-Fe complex. ${ }^{5}$ Salen-Zr complexes catalyzed enantioselective $\alpha$-hydroxylation of $\beta$-ketones esters using cumene hydroperoxide (CHP) as the oxidant in excellent yields

${ }^{a}$ College of Chemistry, Chemical Engineering and Materials Science, Dushu Lake Campus, Soochow University, Suzhou 215123, People's Republic of China. E-mail: zhaobei@suda.edu.cn

${ }^{b}$ Key Laboratory of Organic Synthesis of Jiangsu Province, People's Republic of China. E-mail: yaoym@suda.edu.cn; Fax: +86 51265880305; Tel: +86 51265880305

$\dagger$ Electronic supplementary information (ESI) available. CCDC 1888891 (for 7e) contains the supplementary crystallographic data for this paper. For ESI and crystallographic data in CIF or other electronic format see DOI: 10.1039/c9ra01529a and enantioselectivities. ${ }^{6}$ Epoxidation of non-functionalised alkenes catalyzed by $\mathrm{Mn}$-salen was investigated by Vyas. ${ }^{7}$

However, to the best of our knowledge, examples of highly active lanthanide complexes bearing chiral salen ligands are limited, and their applications are restricted to ring-opening reaction of epoxides, ${ }^{8}$ aminoalkene hydroamination/cyclisation, ${ }^{9}$ and nitro-mannich reaction. ${ }^{10}$ Shibasaki developed asymmetric epoxidation of the electron-deficient $\mathrm{C}=\mathrm{C}$ bonds in enones, $\alpha, \beta$-unsaturated amides and esters, using the combination of lanthanide-BINOL- $\mathrm{Ph}_{3} \mathrm{As}=\mathrm{O}$ as catalyst. ${ }^{11}$ Some lanthanide-BINOL and its derivatives have also been investigated in such a transformation, which gave rise to high enantioselectivities in many cases. ${ }^{12}$ Feng employed $\operatorname{Sc}(\mathrm{OTf})_{3} / N, N^{\prime}-$ dioxide as catalyst to realize the asymmetric epoxidation of $\alpha, \beta$ unsaturated ketones with excellent enantioselectivities. ${ }^{\mathbf{1 3}}$ Recently, our group reported a series of rare-earth metal complexes together with phenoxy-functionalized chiral prolinols which are highly efficient catalysts in the epoxidation of $\alpha, \beta$-unsaturated ketones. Both bisubstituted and trisubstituted chalcones produced the corresponding epoxides in excellent yields (up to 99\%) and enantioselectivities (up to 99\%) using tert-butylhydroperoxide (TBHP) as the oxidant. ${ }^{14}$ As a continuation of our research on the lanthanide-mediated asymmetric transformation, we herein report rare-earth metal complexes bearing chiral salen ligands and their catalytic potential in the enantioselective epoxidation of $\alpha, \beta$-unsaturated ketones.

\section{Results and discussion}

To test the reactivity and selectivity of lanthanide amides in combination with chiral salen ligands, five amides Ln $\left[\mathrm{N}\left(\mathrm{SiMe}_{3}\right)_{2}\right]_{3}(\mathrm{Ln}=\mathrm{Nd}(\mathbf{1}), \mathrm{Sm}(2), \mathrm{Eu}(3), \mathrm{Yb}(4), \mathrm{La}(5))$, two series of phenoxy-functionalized chiral salen ligands $\left[(S, S)-N, N^{\prime}-\right.$ di-(3- $\mathrm{R}^{1}-5-\mathrm{R}^{2}$-salicylidene)-1,2-cyclohexanediamine, $\left[\mathrm{R}^{1}=\mathrm{R}^{2}=\right.$ 
$\operatorname{Me}\left(\mathrm{H}_{2} \mathrm{~L}^{1}\right) ; \mathrm{R}^{1}=\mathrm{R}^{2}={ }^{t} \mathrm{Bu}\left(\mathrm{H}_{2} \mathrm{~L}^{2}\right) ; \mathrm{R}^{1}=\mathrm{H}, \mathrm{R}^{2}=\mathrm{Cl}\left(\mathrm{H}_{2} \mathrm{~L}^{3}\right) ; \mathrm{R}^{1}=\mathrm{H}$; $\mathrm{R}^{2}={ }^{t} \mathrm{Bu}\left(\mathrm{H}_{2} \mathrm{~L}^{4}\right) ; \mathrm{R}^{1}={ }^{t} \mathrm{Bu}, \mathrm{R}^{2}=\mathrm{H}\left(\mathrm{H}_{2} \mathrm{~L}^{5}\right) ; \mathrm{R}^{1}=\mathrm{Me}, \mathrm{R}^{2}=\mathrm{H}$ $\left(\mathrm{H}_{2} \mathrm{~L}^{6}\right) ; \mathrm{R}^{1}={ }^{t} \mathrm{Bu} ; \mathrm{R}^{2}=1$-adam $\left.\left(\mathrm{H}_{2} \mathrm{~L}^{7}\right)\right]$ and $(S, S)-N, N^{\prime}-\left(3-\mathrm{R}^{1}-5-\mathrm{R}^{2}-\right.$ salicylidene)-1,2-diphenyl-1,2-ethanediamine $\left[\mathrm{R}^{1}=\mathrm{R}^{2}=\mathrm{Me}\right.$ $\left.\left(\mathrm{H}_{2} \mathrm{~L}^{8}\right) ; \mathrm{R}^{1}=\mathrm{R}^{2}={ }^{t} \mathrm{Bu}\left(\mathrm{H}_{2} \mathrm{~L}^{9}\right)\right]$ were synthesized according to the previous study. ${ }^{15}$ With the neodymium amide $\mathbf{1}$ and the salen ligand $\mathrm{H}_{2} \mathrm{~L}^{1}$ in hand, the epoxidation reaction of chalcone was carried out in the presence of TBHP in THF. The results are listed in Table 1 . The model reaction underwent quantitatively with unsatisfying enantioselectivity ( $99 \%$ yield and $31 \%$ ee). On the basis of this finding, the influence of the chiral salen ligands on enantioselectivity was carefully investigated via the modification of the phenol moiety. The yields of the corresponding epoxides remained excellent and the ee values varied from 11$31 \%$ (Table 1, entries 1-7). If the chiral linkage was changed to $(S, S)$-1,2-diphenyl-1,2-ethanediamine $\left(\mathrm{H}_{2} \mathrm{~L}^{8}\right.$ and $\left.\mathrm{H}_{2} \mathrm{~L}^{9}\right)$, no significant improvement is observed (Table 1, entries 1-9).

Thus, the chiral salen ligand $\mathrm{H}_{2} \mathrm{~L}^{1}$ was the optimal choice for the model reaction considering both yield and enantioselectivity. Lanthanide amides of different metal centers were

Table 1 Optimization of the reaction conditions ${ }^{a}$
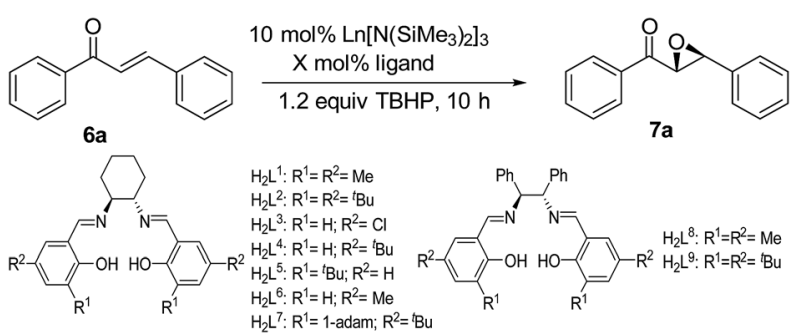

\begin{tabular}{|c|c|c|c|c|c|c|c|c|}
\hline Entry & Cat & $n$ & Ligand & $X$ & Solvent & $T /{ }^{\circ} \mathrm{C}$ & Yield $^{b}(\%)$ & $\mathrm{ee}^{c}(\%)$ \\
\hline 1 & Nd-1 & 10 & $\mathrm{H}_{2} \mathrm{~L}^{1}$ & 12 & THF & $\mathrm{rt}$ & 99 & 31 \\
\hline 2 & Nd-1 & 10 & $\mathrm{H}_{2} \mathrm{~L}^{2}$ & 12 & THF & $\mathrm{rt}$ & 99 & 23 \\
\hline 3 & Nd-1 & 10 & $\mathrm{H}_{2} \mathrm{~L}^{3}$ & 12 & THF & $\mathrm{rt}$ & 86 & 31 \\
\hline 4 & Nd-1 & 10 & $\mathrm{H}_{2} \mathrm{~L}^{4}$ & 12 & THF & $\mathrm{rt}$ & 99 & 27 \\
\hline 5 & Nd-1 & 10 & $\mathrm{H}_{2} \mathrm{~L}^{5}$ & 12 & THF & $\mathrm{rt}$ & 99 & 30 \\
\hline 6 & $\mathrm{Nd}-1$ & 10 & $\mathrm{H}_{2} \mathrm{~L}^{6}$ & 12 & THF & $\mathrm{rt}$ & 99 & 27 \\
\hline 7 & Nd-1 & 10 & $\mathrm{H}_{2} \mathrm{~L}^{7}$ & 12 & THF & $\mathrm{rt}$ & 86 & 11 \\
\hline 8 & Nd-1 & 10 & $\mathrm{H}_{2} \mathrm{~L}^{8}$ & 12 & THF & rt & 99 & 13 \\
\hline 9 & Nd-1 & 10 & $\mathrm{H}_{2} \mathrm{~L}^{9}$ & 12 & THF & $\mathrm{rt}$ & 99 & 23 \\
\hline 10 & Sm-2 & 10 & $\mathrm{H}_{2} \mathrm{~L}^{1}$ & 12 & THF & $\mathrm{rt}$ & 99 & 33 \\
\hline 11 & Eu-3 & 10 & $\mathrm{H}_{2} \mathrm{~L}^{1}$ & 12 & THF & $\mathrm{rt}$ & 89 & 35 \\
\hline 12 & Yb-4 & 10 & $\mathrm{H}_{2} \mathrm{~L}^{1}$ & 12 & THF & $\mathrm{rt}$ & 35 & 4 \\
\hline 13 & La-5 & 10 & $\mathrm{H}_{2} \mathrm{~L}^{1}$ & 12 & THF & $\mathrm{rt}$ & 99 & 41 \\
\hline 14 & La-5 & 10 & $\mathrm{H}_{2} \mathrm{~L}^{1}$ & 12 & Tol & $\mathrm{rt}$ & 99 & 5 \\
\hline 15 & La-5 & 10 & $\mathrm{H}_{2} \mathrm{~L}^{1}$ & 12 & Hex & $\mathrm{rt}$ & 87 & 37 \\
\hline 16 & La-5 & 10 & $\mathrm{H}_{2} \mathrm{~L}^{1}$ & 12 & DME & $\mathrm{rt}$ & 89 & 35 \\
\hline 17 & La-5 & 10 & $\mathrm{H}_{2} \mathrm{~L}^{1}$ & 12 & $\mathrm{CH}_{3} \mathrm{CN}$ & $\mathrm{rt}$ & 99 & 57 \\
\hline 18 & La-5 & 10 & $\mathrm{H}_{2} \mathrm{~L}^{1}$ & 12 & $\mathrm{CH}_{3} \mathrm{CN}$ & 0 & 99 & 70 \\
\hline 19 & La-5 & 10 & $\mathrm{H}_{2} \mathrm{~L}^{1}$ & 12 & $\mathrm{CH}_{3} \mathrm{CN}$ & -20 & 99 & 80 \\
\hline 20 & La-5 & 10 & $\mathrm{H}_{2} \mathrm{~L}^{1}$ & 12 & $\mathrm{CH}_{3} \mathrm{CN}$ & -40 & 82 & 56 \\
\hline 21 & La-5 & 10 & $\mathrm{H}_{2} \mathrm{~L}^{1}$ & 15 & $\mathrm{CH}_{3} \mathrm{CN}$ & -20 & 99 & 71 \\
\hline 22 & La-5 & 10 & $\mathrm{H}_{2} \mathrm{~L}^{1}$ & 20 & $\mathrm{CH}_{3} \mathrm{CN}$ & -20 & 99 & 75 \\
\hline 23 & La-5 & 5 & $\mathrm{H}_{2} \mathrm{~L}^{1}$ & 12 & $\mathrm{CH}_{3} \mathrm{CN}$ & -20 & 99 & 57 \\
\hline
\end{tabular}

${ }^{a}$ The reaction was performed with chalcone $(0.3 \mathrm{mmol})$, TBHP $(0.36$ mmol) in $1 \mathrm{~mL}$ of solvent. ${ }^{b}$ HPLC yield. ${ }^{c}$ Determined by chiral HPLC analysis. studied, and the lanthanum amide 5 gave the best result. The ee value of $7 \mathrm{a}$ reached $41 \%$ without sacrificing yield (Table 1, entries 1 and 10-13).

To further improve enantioselectivity, different solvents were screened. Fortunately, when acetonitrile was used as the solvent in the model reaction, the ee value increased to $57 \%$, while the yield of $99 \%$ was maintained (Table 1, entries 13-17). Some successful cases in the asymmetric epoxidation reactions also achieved high ee values in acetonitrile. ${ }^{14 a, 16}$ Perhaps the weak coordination of acetonitrile to the central lanthanide metal changes the coordination environment. And it leads to better match for the catalyst and substrate, which may plays an important role in the metal-based catalytic asymmetric reaction. A relatively low temperature is usually helpful to improve the enantioselectivity of the asymmetric reaction. To our delight, attempts to lower the reaction temperature have a positive effect on the enantioselectivity. The ee value significantly increased when the reaction temperature decreased, and the $80 \%$ ee of the epoxide $7 \mathrm{a}$ was obtained at $-20{ }^{\circ} \mathrm{C}$ (Table 1 , entries 17-20). Finally, the ratio of the lanthanum amide 5 to the chiral salen ligand $\mathrm{H}_{2} \mathrm{~L}^{1}$ was studied in $\mathrm{CH}_{3} \mathrm{CN}$ and the results showed that the $1: 1.2$ molar ratio was optimal for the model reaction (Table 1, entries 19, 21-22). Thus, $10 \mathrm{~mol} \%$ of La amide 5 in the combination of $12 \mathrm{~mol} \%$ of chiral salen ligand $\mathrm{H}_{2} \mathrm{~L}^{1}$ was an optimal catalytic system for the asymmetric epoxidation of chalcone with TBHP in $\mathrm{CH}_{3} \mathrm{CN}$ at $-20{ }^{\circ} \mathrm{C}$.

Subsequently, various $\alpha, \beta$-unsaturated ketones were synthesized and investigated, and the results are summarized in Chart 1. Most of the disubstituted $\alpha, \beta$-unsaturated ketones underwent the transformation to give epoxides in excellent yields (92-99\%) and good to high enantioselectivities (57-83\%), with the exception of $\mathbf{7 h}(37 \%$ ee) (Chart 1, 7a-7o). Delightedly, much better enantioselectivities, ranging from $80-87 \%$, were observed in reactions of trisubstituted $\alpha, \beta$-unsaturated ketones, which are in general bulky and challenging substrates (Chart $1,7 \mathbf{p}-7 \mathbf{y}$ ). It is noteworthy that these substrates are not only trisubstituted, but also cyclic with restricted mobility, hence more rigid transition states are supposed to generate, which may play a critical role in controlling the enantioselectivity. Relatively high results include the lanthanide-BINOL and its derivatives by Shibasaki, ${ }^{11}$ Inanaga ${ }^{12 a}$ and Ding, ${ }^{12 b}$ and $\mathrm{Sc}(\mathrm{OTf})_{3} / N, N^{\prime}$-dioxide catalyst by Feng, ${ }^{13}$ and rare-earth metal complexes together with phenoxyfunctionalized chiral prolinols reported by us. ${ }^{\mathbf{1 4}}$

The real active species in the current system was investigated. La amide 5 was treated with 1.2 equiv. chiral salen ligand $\mathrm{H}_{2} \mathrm{~L}^{1}$ in THF. After workup, complex 8 was isolated as yellow crystals (Scheme 1). Its ${ }^{1} \mathrm{H}$ NMR spectrum shows absence of a peak at $0.1980 \mathrm{ppm}$, which is ascribed to the coordinated $-\mathrm{N}\left(\mathrm{SiMe}_{3}\right)_{2}$ group. Signals at 3.87-3.55, 1.60 and $1.02 \mathrm{ppm}$ are assigned to cyclohexyl linkage. The peaks at 8.10, 7.91, 7.63, and 7.57 ppm are ascribed to the $\mathrm{CH}=\mathrm{N}$ double bond. These observations prove the formation of complex 8 bearing the chiral salen ligand. Finally, the ratio of the lanthanum atom to chiral salen ligand is determined to be $2: 3$, based on results of elemental analysis and complex titration.

To find out whether complex $\mathbf{8}$ is the active species, it was tested in the model asymmetric epoxidation of chalcone under 


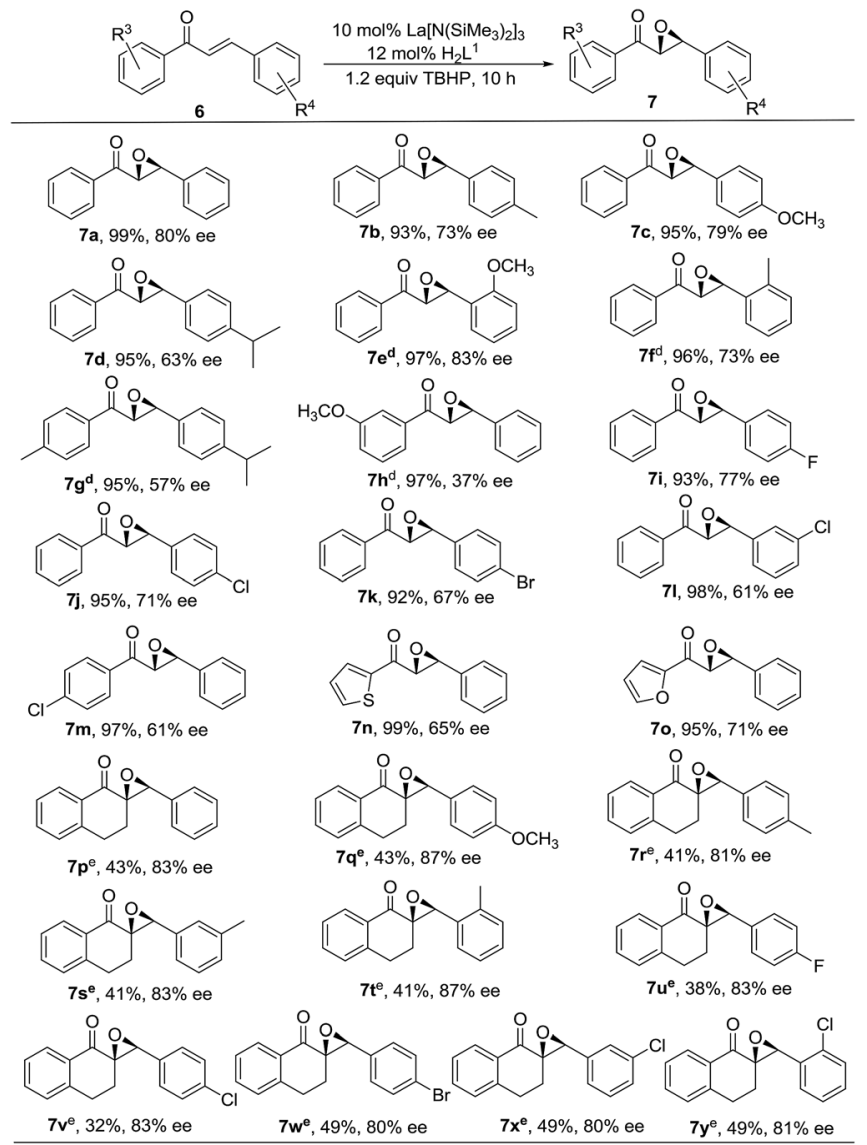

Chart 1 Epoxidation of $\alpha, \beta$-unsaturated ketones catalyzed by

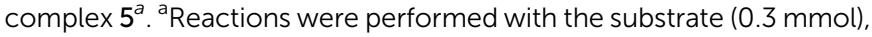
TBHP $(0.36 \mathrm{mmol})$ in $1 \mathrm{~mL} \mathrm{CH} 3 \mathrm{CN}$ at $-20^{\circ} \mathrm{C}$ for $10 \mathrm{~h}$; isolated yield; ee value were determined by chiral HPLC analysis. ${ }^{\mathrm{b}} 15 \mathrm{~h} .{ }^{\mathrm{c}} 48 \mathrm{~h}$.
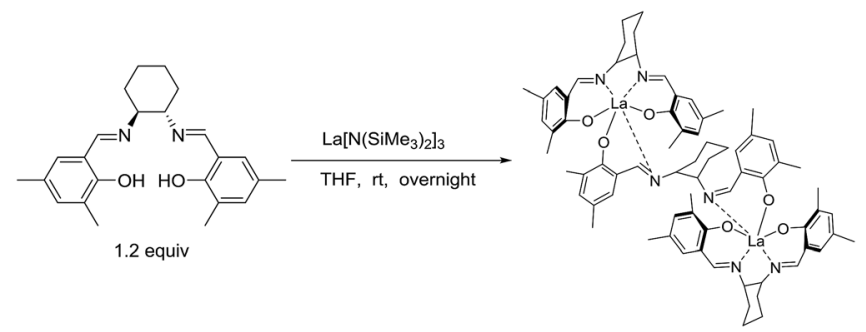

Scheme 1 The preparation of complex 8 .

standard conditions. However, neither the yield nor ee value was comparable with results of in situ generated catalyst. The target epoxide was obtained in $91 \%$ yield and only $70 \%$ ee (Table 2, entry 1). Comparing described catalyst system with complex 8, the difference is $\mathrm{HN}\left(\mathrm{SiMe}_{3}\right)_{2}$ generated in situ in the former, which may have the positive effects on the asymmetric transformation. To verify the hypothesis, complex 8 together with $20 \mathrm{~mol} \% \mathrm{HN}\left(\mathrm{SiMe}_{3}\right)_{2}$ were added to the model reaction. The outcome of $98 \%$ yield with $78 \%$ ee indicates that the addition of $\mathrm{HN}\left(\mathrm{SiMe}_{3}\right)_{2}$ indeed has a positive effect on the asymmetric catalytic process (Table 2, entry 2). Screening of
Table 2 The effect of additives on the asymmetric epoxidation of chalcone $^{a}$

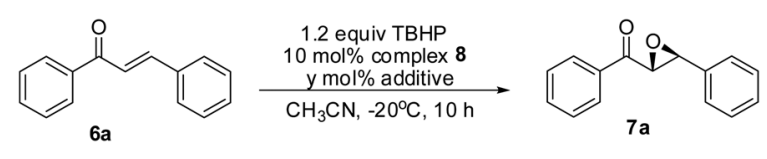

\begin{tabular}{lllll}
\hline Entry & Additive & $Y$ & Yeild $^{b}(\%)$ & ee $^{c}(\%)$ \\
\hline 1 & - & 20 & 91 & 70 \\
2 & $\mathrm{HN}\left(\mathrm{SiMe}_{3}\right)_{2}$ & 20 & 98 & 78 \\
3 & $\mathrm{DBU}$ & 20 & 93 & 15 \\
4 & Pyridine & 20 & 88 & 59 \\
5 & $\mathrm{Et}_{3} \mathrm{~N}$ & 20 & 86 & 60 \\
6 & $2,2-$ Bipyridine $_{2}$ & 20 & 85 & 56 \\
7 & $\mathrm{HN}\left(\mathrm{SiMe}_{3}\right)_{2}$ & 10 & 93 & 73 \\
8 & $\mathrm{HN}\left(\mathrm{SiMe}_{3}\right)_{2}$ & 30 & 95 & 75 \\
9 & $\mathrm{HN}\left(\mathrm{SiMe}_{3}\right)_{2}$ & 50 & 90 & 66
\end{tabular}

${ }^{a}$ Reactions were performed with chalcone $(0.3 \mathrm{mmol})$, TBHP $(0.36$ mmol), $1 \mathrm{~mL} \mathrm{CH}_{3} \mathrm{CN} .{ }^{b}$ HPLC yield. ${ }^{c}$ Determined by chiral HPLC analysis.

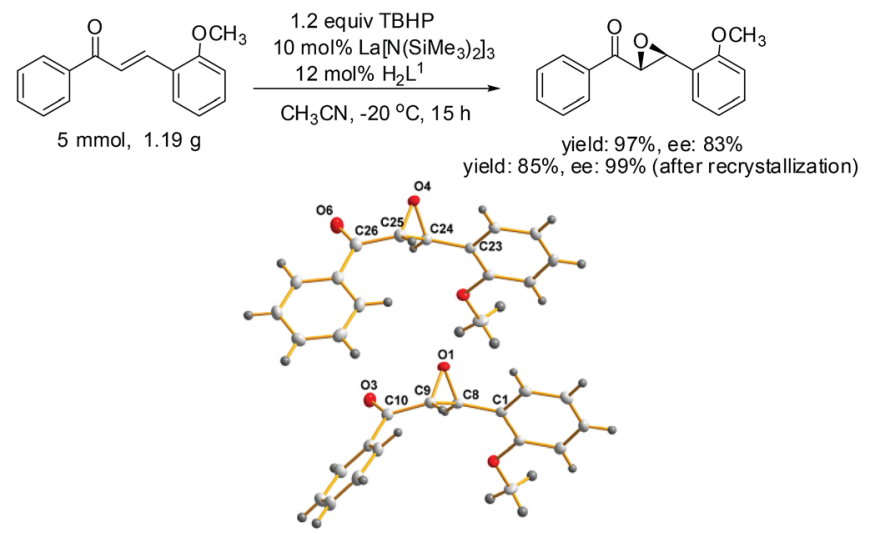

Scheme 2 The synthesis and the molecular structure of compound 7e.

basic additives, including 1,8-diazabicyclo[5,4,0]undec-7-ene (DBU), $\mathrm{Et}_{3} \mathrm{~N}$, pyridine, and 2,2'-bipyridine, proved that $\mathrm{HN}\left(\mathrm{SiMe}_{3}\right)_{2}(20 \mathrm{~mol} \%)$ is the optimal choice (Table 2, entries 29).

Finally, the outcome of the model reaction on a gram scale was satisfactory with an appropriate prolonging of the reaction time and the absolute configuration of the epoxides was determined by single crystal diffraction analysis, chiral HPLC and optical rotation analysis of a representative crystal $7 e$ (Scheme 2).

The molecular structure of $7 \mathrm{e}$ showing $20 \%$ probability ellipsoids. Selected bond lengths $(\AA)$ and bond angles $\left({ }^{\circ}\right)$ : $\mathrm{O}(1)-$ $\mathrm{C}(8)$ 1.4235(3), O(1)-C(9) 1.430(4), C(8)-C(9) 1.494(4), O(4)-C(24) 1.435(4), $\mathrm{O}(4)-\mathrm{C}(25)$ 1.415(4), $\mathrm{C} 24-\mathrm{C} 25$ 1.482(4), $\mathrm{O}(1)-\mathrm{C}(8)-\mathrm{C}(1)$ 116.6(2), O(1)-C(9)-C(10) 115.1(3), O(1)-O(8)-C(9) 58.37(18), $\mathrm{O}(1)-\mathrm{C}(9)-\mathrm{C}(8)$ 58.74(18), C(9)-O(1)-C(8) 62.89(19), O(4)-C(24)$\mathrm{C}(23)$ 116.1(2), $\mathrm{O}(4)-\mathrm{C}(25)-\mathrm{C}(26)$ 113.8(3), $\mathrm{O}(4)-\mathrm{C}(24)-\mathrm{C}(25)$ 58.02(19), O(4)-C(25)-C(24) 59.33(19), C(24)-O(4)-C(25) 62.6(2). 


\section{Conclusions}

In summary, the asymmetric epoxidation of $\alpha, \beta$-unsaturated ketones was catalyzed by a new series of lanthanide amides with chiral salen ligands. After careful screening, $10 \mathrm{~mol} \%$ of the La amide $\mathrm{La}\left[\mathrm{N}\left(\mathrm{SiMe}_{3}\right)_{2}\right]_{3}$ together with the chiral salen ligand $(S, S)$ $N, N^{\prime}$-(3,5-dimethylsalicylidene)-1,2-cyclohexanediamine in a 1 : 1.2 molar ratio were found to be the optimal catalyst in $\mathrm{CH}_{3} \mathrm{CN}$ at $-20{ }^{\circ} \mathrm{C}$. For the disubstituted chalcone derivatives, the target epoxides were obtained in excellent yields (92-99\%) with moderate to good ee values (57-83\%), while epoxides from the trisubstituted $\alpha, \beta$-unsaturated ketones were generated in high enantioselectivities (80-87\%) and relative low yields (32$57 \%$ ). Complex 8 resulting from reaction of lanthanum amide and salen ligand was isolated and characterized, which, together with $\mathrm{HN}\left(\mathrm{SiMe}_{3}\right)_{2}$ additive, gave comparable catalytic result with that of in situ generated catalyst. The absolute configuration of the target chiral epoxides was determined by single crystal diffraction analysis.

\section{Experimental}

\section{General information}

All manipulations and reactions involving air and water sensitive components were performed with the standard Schlenk techniques. Solvents, such as THF, toluene and hexane, were degassed and distilled from sodium benzophenone ketyl under argon before use. Analytical thin layer chromatography (TLC) was performed using F254 pre-coated silica gel plate $(0.2 \mathrm{~mm}$ thickness). After elution, plates were detected using UV radiation $(254 \mathrm{~nm})$ on a UV lamp. Flash chromatography was performed using 200-300 mesh silica gel with freshly distilled solvents. Nuclear magnetic resonance spectra were obtained on a Bruker AV-400 apparatus ( $\mathrm{CDCl}_{3}$ as solvent). High Resolution Mass (HRMS) spectra were obtained using Bruker ESI-TOF. Rare-earth metal analysis was performed by EDTA titration with a xylenol orange indicator and a hexamine buffer. Carbon, hydrogen and nitrogen analyses were performed by direct combustion with a Carlo-Erba EA-1110 instrument. The ee values determination was carried out using HPLC (Agilent Technologies 1200 Series) with Daicel chiralcel columns at room temperature. Optical rotation was measured using an Autopol IV polarimeter equipped with a sodium vapor lamp at $589 \mathrm{~nm}$. The absolute configuration of 7e was determined by the detection of single crystal diffraction. Intensity data were collected with a Rigaku Mercury CCD area detector in $\omega$ scan mode using $\mathrm{Cu}-\mathrm{K} \alpha$ radiation $(\lambda=1.54178 \AA)$. Hence, the absolute configurations of $7 \mathbf{a}-7 \mathbf{y}$ were assigned by analogy, assuming the same reaction pathway and the same analysis.

\section{Synthesis of lanthanum complex 8}

Under a standard Schlenk vacuum line, to a THF solution of $4 \mathrm{mmol} \mathrm{La}\left[\mathrm{N}\left(\mathrm{SiMe}_{3}\right)_{2}\right]_{3}, 6 \mathrm{mmol} \mathrm{H}_{2} \mathrm{~L}^{1}$ in $4 \mathrm{~mL}$ THF was added dropwise at room temperature. The mixture was continued to stir overnight. Then, removing the THF solvent in vacuo, the crude product was washed with hexane for three times and a faint yellow solid was obtained by centrifugal separation. Finally, complex $\mathbf{8}$ was purified by recrystallization in the mixed solvent of toluene and hexane.

\section{General procedure for the synthesis of the substituted epoxides $7 \mathbf{a}-7 \mathbf{y}$}

Under argon atmosphere, lanthanum amide $\mathrm{La}\left[\mathrm{N}\left(\mathrm{SiMe}_{3}\right)_{2}\right]_{3}$ (0.03 mmol, $18.65 \mathrm{mg}$ ) was added to a solution of $\mathrm{H}_{2} \mathrm{~L}^{1}$ $(0.036 \mathrm{mmol}, 13.62 \mathrm{mg})$ in $\mathrm{CH}_{3} \mathrm{CN}(1 \mathrm{~mL})$. After stirring for $30 \mathrm{~min}$, unsaturated ketones $(0.3 \mathrm{mmol})$ was added and stirred for further $30 \mathrm{~min}$. After that, oxidant TBHP $(0.36 \mathrm{mmol})$ was added to the mixture. The system was stirred for $10 \mathrm{~h}$ at $-20^{\circ} \mathrm{C}$. Quenched by the saturated solution of $\mathrm{Na}_{2} \mathrm{SO}_{3}$, the crude product was extracted by ethyl acetate, then purified by column chromatography (ethyl acetate-petroleum ether, $1: 10$ ) to obtain the target epoxide. The enantiomeric excess of epoxide was determined by chiral HPLC analysis.

\section{Spectroscopic data for ligands}

$(S, S)-N, N^{\prime}$-Di(3,5-dimethylsalicylidene)-1,2-cyclohexanediamine $\left(\mathbf{H}_{2} \mathbf{L}^{\mathbf{1}}\right)$. A yellow solid; yield: $70 \%$; $[\alpha]_{\mathrm{D}}^{25}-255^{\circ}\left(c 1.0\right.$ in $\left.\mathrm{CHCl}_{3}\right) ;{ }^{1} \mathrm{H}$ NMR (400 MHz, $\left.\mathrm{CDCl}_{3}\right) \delta 13.39$ (s, 2H, OH), 8.20 (s, 2H, CH), 6.94 (s, 2H, Ar-H), 6.80 (d, J=1.5 Hz, 2H, Ar-H), 3.29 (m, 2H, CH), 2.20 $\left(\mathrm{d}, J=6.7 \mathrm{~Hz}, 12 \mathrm{H}, \mathrm{CH}_{3}\right), 1.88\left(\mathrm{~m}, 4 \mathrm{H}, \mathrm{CH}_{2} \mathrm{CH}_{2}\right), 1.70\left(\mathrm{~m}, 2 \mathrm{H}, \mathrm{CH}_{2}\right)$, $1.46\left(\mathrm{t}, J=9.6 \mathrm{~Hz}, 2 \mathrm{H}, \mathrm{CH}_{2}\right) .{ }^{13} \mathrm{C} \mathrm{NMR}\left(100 \mathrm{MHz}, \mathrm{CDCl}_{3}\right) \delta 164.3$, 156.5, 133.7, 128.6, 126.5, 124.8, 117.1, 72.2, 32.7, 23.7, 19.8, 14.9. ppm. HRMS (ESI-MS) calcd for $\mathrm{C}_{24} \mathrm{H}_{31} \mathrm{~N}_{2} \mathrm{O}_{2}[\mathrm{M}+\mathrm{H}]^{+}$: 379.2386, found: 379.2391 .

$(S, S)-N, N^{\prime}$-Di(3,5-di-butylsalicylidene)-1,2-cyclohexanediamine $\left(\mathbf{H}_{2} \mathbf{L}^{2}\right)$. A yellow solid; yield: $80 \%$. $[\alpha]_{\mathrm{D}}^{25}-265^{\circ}\left(c 1.0\right.$ in $\left.\mathrm{CHCl}_{3}\right) \cdot{ }^{1} \mathrm{H}$ NMR (400 MHz, $\left.\mathrm{CDCl}_{3}\right): \delta 13.73(\mathrm{~s}, 2 \mathrm{H}, \mathrm{OH}), 8.32(\mathrm{~s}, 2 \mathrm{H}, \mathrm{CH}), 7.33$ $(\mathrm{d}, J=2.4 \mathrm{~Hz}, 2 \mathrm{H}, \mathrm{Ar}-\mathrm{H}), 7.01$ (d, $J=2.4 \mathrm{~Hz}, 2 \mathrm{H}, \mathrm{Ar}-\mathrm{H}), 3.34(\mathrm{~m}$, $2 \mathrm{H}, \mathrm{CHCH}), 1.93\left(\mathrm{dd}, J=29.2,11.5 \mathrm{~Hz}, 4 \mathrm{H}, \mathrm{CH}_{2} \mathrm{CH}_{2}\right), 1.76(\mathrm{~d}, J=$ $10.0 \mathrm{~Hz}, 2 \mathrm{H}, \mathrm{CH}_{2}$ ), 1.49 (d, $J=9.7 \mathrm{~Hz}, 2 \mathrm{H}, \mathrm{CH}_{2}$ ), 1.44 (s, 18H, $\left.\mathrm{C}\left(\mathrm{CH}_{3}\right)_{3}\right), 1.26\left(\mathrm{~s}, 18 \mathrm{H}, \mathrm{C}\left(\mathrm{CH}_{3}\right)_{3}\right) .{ }^{13} \mathrm{C} \mathrm{NMR}\left(100 \mathrm{MHz}, \mathrm{CDCl}_{3}\right)$ : $\delta$ 165.8, 158.0, 139.90, 136.4, 126.8, 126.1, 117.9, 72.4, 35.0, 34.1, 33.3, 31.4, 29.5, 24.4 ppm. HRMS (ESI-MS) calcd for $\mathrm{C}_{36} \mathrm{H}_{55} \mathrm{~N}_{2} \mathrm{O}_{2}$ $[\mathrm{M}+\mathrm{H}]^{+}: 547.4264$, found: 547.4261 .

$(S, S)-N, N^{\prime}$-Di(5-chlorosalicylidene)-1,2-cyclohexanediamine $\left(\mathbf{H}_{2} \mathbf{L}^{3}\right)$. A yellow solid; yield: $80 \%$. $[\alpha]_{\mathrm{D}}^{25}-195^{\circ}\left(c 1.0\right.$ in $\left.\mathrm{CHCl}_{3}\right)$. ${ }^{1} \mathrm{H}$ NMR (400 MHz, $\left.\mathrm{CDCl}_{3}\right): \delta 13.20(\mathrm{~s}, 2 \mathrm{H}, \mathrm{OH}), 8.18(\mathrm{~s}, 2 \mathrm{H}$, $\mathrm{CH}), 7.19(\mathrm{dd}, J=8.8,2.6 \mathrm{~Hz}, 2 \mathrm{H}, \mathrm{Ar}-\mathrm{H}), 7.12(\mathrm{~d}, J=2.6 \mathrm{~Hz}, 2 \mathrm{H}$, $\mathrm{Ar}-\mathrm{H}), 6.84$ (d, J = 8.8 Hz, 2H, Ar-H), 3.32 (m, 2H, CHCH), 1.91 (dd, $\left.J=18.6,9.3 \mathrm{~Hz}, 4 \mathrm{H}, \mathrm{CH}_{2} \mathrm{CH}_{2}\right), 1.72\left(\mathrm{~m}, 2 \mathrm{H}, \mathrm{CH}_{2}\right), 1.48$ (t, $J$ $\left.=10.0 \mathrm{~Hz}, 2 \mathrm{H}, \mathrm{CH}_{2}\right) .{ }^{13} \mathrm{C} \mathrm{NMR}\left(100 \mathrm{MHz} \mathrm{CDCl}_{3}\right): \delta 163.6$, 159.5, 132.2, 130.6, 123.3, 119.3, 118.4, 72.7, 32.9, 24.1 ppm. HRMS (ESI-MS) calcd for $\mathrm{C}_{20} \mathrm{H}_{21} \mathrm{ClN}_{2} \mathrm{O}_{2}[\mathrm{M}+\mathrm{H}]^{+}$: 391.0980, found: 391.0987 .

$(S, S)-N, N^{\prime}$-Di(5-tert-butylsalicylidene)-1,2-cyclohexanediamine $\left(\mathbf{H}_{2} \mathbf{L}^{\mathbf{4}}\right)$. A yellow solid; yield: $72 \%$. $[\alpha]_{\mathrm{D}}^{25}-175^{\circ}\left(c 1.0\right.$ in $\left.\mathrm{CHCl}_{3}\right) \cdot{ }^{1} \mathrm{H}$ NMR (400 MHz, $\left.\mathrm{CDCl}_{3}\right): \delta 13.13(\mathrm{~s}, 2 \mathrm{H}), 8.25(\mathrm{~s}, 2 \mathrm{H}, \mathrm{OH}), 7.29(\mathrm{~m}$, $2 \mathrm{H}, \mathrm{Ar}-\mathrm{H}), 7.12$ (d, $J=2.5 \mathrm{~Hz}, 2 \mathrm{H}, \mathrm{Ar}-\mathrm{H}), 6.83$ (d, $J=8.6 \mathrm{~Hz}, 2 \mathrm{H}$, $\mathrm{Ar}-\mathrm{H}$ ), 3.31 (m, 2H, CHCH), 1.89 (dd, $J=18.0,9.9 \mathrm{~Hz}, 4 \mathrm{H}$, $\mathrm{CH}_{2} \mathrm{CH}_{2}$ ), 1.72 (dd, $J=20.6,9.8 \mathrm{~Hz}, 2 \mathrm{H}, \mathrm{CH}_{2}$ ), 1.47 (t, $J=10.1 \mathrm{~Hz}$, $\left.2 \mathrm{H}, \mathrm{CH}_{2}\right), 1.24\left(\mathrm{~s}, 18 \mathrm{H}, \mathrm{C}\left(\mathrm{CH}_{3}\right)_{3}\right) \cdot{ }^{13} \mathrm{C} \mathrm{NMR}\left(100 \mathrm{MHz}, \mathrm{CDCl}_{3}\right)$ : $\delta 165.0,158.6,141.2,129.5,127.9,118.0,116.3,72.8,33.9,33.2$, 
31.4, 24.2 ppm. HRMS (ESI-MS) calcd for $\mathrm{C}_{28} \mathrm{H}_{39} \mathrm{~N}_{2} \mathrm{O}_{2}[\mathrm{M}+\mathrm{H}]^{+}$: 435.3021, found: 435.3028 .

$(S, S)$ - $N, N^{\prime}$-Di(3-tert-butylsalicylidene)-1,2-cyclohexanediamine $\left(\mathbf{H}_{2} \mathbf{L}^{5}\right)$. A yellow solid; yield: $72 \%$. $[\alpha]_{\mathrm{D}}^{25}-398^{\circ}\left(c 1.0\right.$ in $\left.\mathrm{CHCl}_{3}\right) \cdot{ }^{1} \mathrm{H}$ NMR (400 MHz, $\left.\mathrm{CDCl}_{3}\right): \delta 13.82(\mathrm{~s}, 2 \mathrm{H}, \mathrm{OH}), 8.22(\mathrm{~s}, 2 \mathrm{H}, \mathrm{CH}), 7.17$ (dd, $J=7.9,1.5 \mathrm{~Hz}, 2 \mathrm{H}, \mathrm{Ar}-\mathrm{H}), 6.92(\mathrm{dd}, J=7.6,1.4 \mathrm{~Hz}, 2 \mathrm{H}, \mathrm{Ar}-\mathrm{H})$, $6.64(\mathrm{t}, J=7.6 \mathrm{~Hz}, 2 \mathrm{H}, \mathrm{Ar}-\mathrm{H}), 3.25(\mathrm{~m}, 2 \mathrm{H}, \mathrm{CHCH}), 1.91(\mathrm{~d}, J=$ $\left.13.9 \mathrm{~Hz}, 2 \mathrm{H}, \mathrm{CH}_{2}\right), 1.81\left(\mathrm{~m}, 2 \mathrm{H}, \mathrm{CH}_{2}\right), 1.68(\mathrm{dd}, J=22.0,11.4 \mathrm{~Hz}$, $\left.2 \mathrm{H}, \mathrm{CH}_{2}\right), 1.40\left(\mathrm{~m}, 2 \mathrm{H}, \mathrm{CH}_{2}\right), 1.33\left(\mathrm{~s}, 18 \mathrm{H}, \mathrm{C}\left(\mathrm{CH}_{3}\right)_{3}\right) \cdot{ }^{13} \mathrm{C} \mathrm{NMR}(100$ $\left.\mathrm{MHz}, \mathrm{CDCl}_{3}\right): \delta 164.9,159.3,133.2,129.2,125.7,118.0,72.6,33.2$, 24.2, 15.5 ppm. HRMS (ESI-MS) calcd for $\mathrm{C}_{28} \mathrm{H}_{39} \mathrm{~N}_{2} \mathrm{O}_{2}[\mathrm{M}+\mathrm{H}]^{+}$: 435.3021, found: 435.30323 .

$(S, S)-N, N^{\prime}$-Di(5-methylsalicylidene)-1,2-cyclohexanediamine $\left(\mathbf{H}_{2} \mathbf{L}^{6}\right)$. A yellow solid; yield: $71 \% \cdot[\alpha]_{\mathrm{D}}^{25}-495^{\circ}(c 1.0$ in $\mathrm{CHCl} 3)$. 1H NMR (400 MHz, CDCl3): $\delta 13.63(\mathrm{~s}, 2 \mathrm{H}, \mathrm{OH}), 8.27(\mathrm{~s}, 2 \mathrm{H}$, $\mathrm{CH}), 7.12(\mathrm{~d}, \mathrm{~J}=7.3 \mathrm{~Hz}, 2 \mathrm{H}, \mathrm{Ar}-\mathrm{H}), 7.01$ (d, $J=7.6 \mathrm{~Hz}, 2 \mathrm{H}, \mathrm{Ar}-$ $\mathrm{H}), 6.72(\mathrm{t}, J=7.5 \mathrm{~Hz}, 2 \mathrm{H}, \mathrm{Ar}-\mathrm{H}), 3.31(\mathrm{~m}, 2 \mathrm{H}, \mathrm{CH}), 2.24(\mathrm{~s}, 6 \mathrm{H}$, $\mathrm{H}_{3}$ ), $1.91\left(\mathrm{~m}, 4 \mathrm{H}, \mathrm{CH}_{2} \mathrm{CH}_{2}\right.$ ), 1.71 (d, $\left.J=10.1 \mathrm{~Hz}, 2 \mathrm{H}, \mathrm{CH}_{2}\right), 1.48$ (dd, $J=12.7,6.1 \mathrm{~Hz}, 2 \mathrm{H}, \mathrm{CH}_{2}$ ). ${ }^{13} \mathrm{C} \mathrm{NMR}\left(100 \mathrm{MHz}, \mathrm{CDCl}_{3}\right.$ ): $\delta$ 164.4, 158.8, 132.7, 128.7, 125.2, 117.5, 72.1, 32.7, 23.7, 15.0 ppm. HRMS (ESI-MS) calcd for $\mathrm{C}_{22} \mathrm{H}_{27} \mathrm{~N}_{2} \mathrm{O}_{2}[\mathrm{M}+\mathrm{H}]^{+}$: 351.2073, found: 351.2077 .

$(S, S)-N, N^{\prime}$-Di(5-methyl-3-adamantylsalicylidene)-1,2-cyclohexanediamine $\left(\mathbf{H}_{2} \mathbf{L}^{7}\right)$. A yellow solid; yield: $45 \%$. $[\alpha]_{\mathrm{D}}^{25}-235^{\circ}(c 1.0$ in $\mathrm{CHCl}_{3}$ ). ${ }^{1} \mathrm{H}$ NMR (400 MHz, $\mathrm{CDCl}_{3}$ ): $\delta 13.66$ (s, 2H, OH), 8.30 (s, $2 \mathrm{H}, \mathrm{CH}), 7.24$ (d, $J=2.4 \mathrm{~Hz}, 2 \mathrm{H}, \mathrm{Ar}-\mathrm{H}), 6.97$ (d, $J=2.4 \mathrm{~Hz}, 2 \mathrm{H}, \mathrm{Ar}-$ $\mathrm{H}), 3.31(\mathrm{~m}, 2 \mathrm{H}, \mathrm{CH}), 2.15\left(\mathrm{~s}, 12 \mathrm{H}, \mathrm{CH}_{2}\right), 2.07(\mathrm{~s}, 6 \mathrm{H}, \mathrm{CH}), 1.90(\mathrm{~m}$, $\left.4 \mathrm{H}, \mathrm{CH}_{2} \mathrm{CH}_{2}\right), 1.79\left(\mathrm{~s}, 12 \mathrm{H}, \mathrm{CH}_{2}\right), 1.45\left(\mathrm{~m}, 2 \mathrm{H}, \mathrm{CH}_{2}\right), 1.30(\mathrm{~m}, 2 \mathrm{H}$, $\mathrm{CH}_{2}$ ), $1.24\left(\mathrm{~s}, 18 \mathrm{H}, \mathrm{CH}_{3}\right) \cdot{ }^{13} \mathrm{C} \mathrm{NMR}\left(100 \mathrm{MHz}, \mathrm{CDCl}_{3}\right): \delta$ 165.0, 158.6, 141.2, 129.5, 127.9, 118.0, 116.2, 72.8, 33.9, 33.2, 31.40, 24.2 ppm. HRMS (ESI-MS) calcd for $\mathrm{C}_{48} \mathrm{H}_{67} \mathrm{~N}_{2} \mathrm{O}_{2}[\mathrm{M}+\mathrm{H}]^{+}$: 703.5203, found: 703.5204.

$(S, S)-N, N^{\prime}$-Di(3,5-dimethylsalicylidene)-1,2-diphenyl-1,2-ethanediamine $\left(\mathbf{H}_{2} \mathbf{L}^{8}\right)$. A yellow solid; yield: $72 \%$. $[\alpha]_{\mathrm{D}}^{25} 189^{\circ}(c 1.0$ in $\mathrm{CHCl}_{3}$ ). ${ }^{1} \mathrm{H} \mathrm{NMR}\left(400 \mathrm{MHz}, \mathrm{CDCl}_{3}\right): \delta 13.25(\mathrm{~s}, 2 \mathrm{H}, \mathrm{OH}), 8.27(\mathrm{~s}, 2 \mathrm{H}$, $\mathrm{CH}), 7.16$ (m, 10H, Ar-H), 6.94 (s, 2H, Ar-H), 6.77 (s, 2H, Ar-H), 4.68 (s, 2H, CHCH), 2.24 (s, 6H, $\left.\mathrm{CH}_{3}\right), 2.17\left(\mathrm{~s}, 6 \mathrm{H}, \mathrm{CH}_{3}\right) .{ }^{13} \mathrm{C} \mathrm{NMR}$ $\left(100 \mathrm{MHz} \mathrm{CDCl}_{3}\right): \delta$ 166.3, 156.9, 139.7, 134.6, 129.5, 128.3, 128.0, 127.5, 127.2, 125.4, 117.6, 80.5, 20.3, 15.5 ppm. HRMS (ESI-MS) calcd for $\mathrm{C}_{32} \mathrm{H}_{33} \mathrm{~N}_{2} \mathrm{O}_{2}[\mathrm{M}+\mathrm{H}]^{+}: 477.2542$, found: 477.2549 .

$(S, S)-N, N^{\prime}$-Di(3,5-di-tert-butylsalicylidene)-1,2-diphenyl-1,2ethanediamine $\left(\mathbf{H}_{2} \mathbf{L}^{9}\right)$. A yellow solid; yield: $81 \%$. $[\alpha]_{\mathrm{D}}^{25} 203^{\circ}$ $\left(c 1.0\right.$ in $\left.\mathrm{CHCl}_{3}\right) .{ }^{1} \mathrm{H}$ NMR (400 MHz, $\left.\mathrm{CDCl}_{3}\right): \delta 13.60(\mathrm{~s}, 2 \mathrm{H}, \mathrm{OH})$, 8.40 (s, 2H, CH), 7.31 (s, 2H, Ar-H), 7.16 (m, 10H, Ar-H), 6.98 (s, $2 \mathrm{H}, \mathrm{Ar}-\mathrm{H}), 4.72(\mathrm{~s}, 2 \mathrm{H}, \mathrm{CHCH}), 1.42\left(\mathrm{~s}, 18 \mathrm{H}, \mathrm{CH}_{3}\right), 1.22(\mathrm{~s}, 18 \mathrm{H}$, $\left.\mathrm{CH}_{3}\right) .{ }^{13} \mathrm{C} \mathrm{NMR}\left(100 \mathrm{MHz}, \mathrm{CDCl}_{3}\right): \delta 167.1,158.0,140.1,139.9$, 136.4, 128.3, 128.1, 127.5, 127.2, 126.4, 117.9, 80.2, 35.1, 34.1, 31.5, 29.5 ppm. HRMS (ESI-MS) calcd for $\mathrm{C}_{44} \mathrm{H}_{57} \mathrm{~N}_{2} \mathrm{O}_{2}[\mathrm{M}+\mathrm{H}]^{+}$: 645.4420, found: 645.4426 .

\section{Spectroscopic data for saymmetric epoxidation products}

$((2 R, 3 S)-3-P h e n y l o x i r a n-2-y l)-p h e n y l m e t h a n o n e \quad 7 a .^{14 a} \quad A$ white powder; yield $99 \%$, ee $80 \% ;[\alpha]_{\mathrm{D}}^{25}-112^{\circ}(c 0.3$ in acetone). ${ }^{1} \mathrm{H}$ NMR (400 MHz, $\left.\mathrm{CDCl}_{3}\right) \delta 8.00(\mathrm{~d}, J=7.3 \mathrm{~Hz}, 2 \mathrm{H}, \mathrm{Ar}-\mathrm{H}), 7.61$ (t, $J=7.4 \mathrm{~Hz}, 1 \mathrm{H}, \mathrm{Ar}-\mathrm{H}), 7.47$ (t, $J=7.7 \mathrm{~Hz}, 2 \mathrm{H}, \mathrm{Ar}-\mathrm{H}), 7.38(\mathrm{~m}$, $5 \mathrm{H}, \mathrm{Ar}-\mathrm{H}), 4.29$ (d, $J=1.7 \mathrm{~Hz}, 1 \mathrm{H}, \mathrm{CH}), 4.07$ (d, $J=1.6 \mathrm{~Hz}, 1 \mathrm{H}$,
CH). HPLC: OD-H column, 90\% hexanes, 10\% ${ }^{\mathrm{i}} \mathrm{PrOH}, 1.0$ $\mathrm{mL} \min ^{-1}, t_{\mathrm{r}}$ (minor) $=7.6 \mathrm{~min}, t_{\mathrm{r}}$ (major) $=8.4 \mathrm{~min}$. HRMS (ESI, $m / z$ ) calcd for $\mathrm{C}_{15} \mathrm{H}_{14} \mathrm{O}_{2} \mathrm{Na}[\mathrm{M}+\mathrm{Na}]^{+}: 247.0735$, found: 247.0738. Mp: $74-76{ }^{\circ} \mathrm{C}$.

((2R,3S)-3-(4-Methphenyl)oxiran-2-yl)-(phenyl)methanone $7 \mathbf{b}^{\mathbf{1 4 a}}$ A white powder; yield $93 \%$, ee $73 \% ;[\alpha]_{\mathrm{D}}^{25}-110^{\circ}$ ( $c 0.3$ in acetone). ${ }^{1} \mathrm{H}$ NMR (400 MHz, $\left.\mathrm{CDCl}_{3}\right) \delta 7.94(\mathrm{~m}, 2 \mathrm{H}, \mathrm{Ar}-\mathrm{H}), 7.55$ (m, 1H, ArH), $7.42(\mathrm{~m}, 2 \mathrm{H}, \mathrm{Ar}-\mathrm{H}), 7.17$ (q, $J=7.8 \mathrm{~Hz}, 5 \mathrm{H}, \mathrm{Ar}-\mathrm{H}), 4.22$ (d, $J=$ $1.9 \mathrm{~Hz}, 1 \mathrm{H}, \mathrm{CH}), 3.97$ (d, J = $1.8 \mathrm{~Hz}, 1 \mathrm{H}, \mathrm{CH}), 2.31\left(\mathrm{~s}, 3 \mathrm{H}, \mathrm{CH}_{3}\right)$. HPLC: OD-H column, 90\% hexanes, $10 \%{ }^{\mathrm{i}} \mathrm{PrOH}, 1.0 \mathrm{~mL} \mathrm{~min}{ }^{-1}, t_{\mathrm{r}}$ (minor) $=6.5 \mathrm{~min}, t_{\mathrm{r}}$ (major) $=7.3 \mathrm{~min}$. HRMS (ESI, $\mathrm{m} / \mathrm{z}$ ) calcd for $\mathrm{C}_{16} \mathrm{H}_{14} \mathrm{O}_{2} \mathrm{Na}[\mathrm{M}+\mathrm{Na}]^{+}: 261.2758$, found: 261.2753. Mp: $76{ }^{\circ} \mathrm{C}$.

((2R,3S)-3-(4-Methoxyphenyl)oxiran-2-yl)-(phenyl)methanone 7c. ${ }^{14 a}$ A white powder; yield $95 \%$, ee $79 \%$; $[\alpha]_{\mathrm{D}}^{25}-102^{\circ}(c 0.3 \mathrm{in}$ acetone). ${ }^{1} \mathrm{H}$ NMR (400 MHz, $\left.\mathrm{CDCl}_{3}\right) \delta 8.01$ (m, 2H, Ar-H), 7.62 (m, 1H, Ar-H), 7.49 (m, 2H, Ar-H), 7.30 (m, 2H, Ar-H), 6.93 (m, $2 \mathrm{H}, \mathrm{Ar}-\mathrm{H}), 4.29$ (d, $J=1.9 \mathrm{~Hz}, 1 \mathrm{H}, \mathrm{CH}), 4.03(\mathrm{~d}, J=1.8 \mathrm{~Hz}, 1 \mathrm{H}$, $\mathrm{CH}$ ), 3.83 (s, 3H, $\mathrm{CH}_{3}$ ). HPLC: OD-H column, 90\% hexanes, $10 \%$ ${ }^{\mathrm{i}} \mathrm{PrOH}, 1.0 \mathrm{~mL} \mathrm{\operatorname {min } ^ { - 1 }}, t_{\mathrm{r}}$ (minor) $=9.4 \mathrm{~min}, t_{\mathrm{r}}$ (major) $=10.4 \mathrm{~min}$. HRMS (ESI, $m / z$ ) calcd for $\mathrm{C}_{16} \mathrm{H}_{14} \mathrm{O}_{2} \mathrm{Na}[\mathrm{M}+\mathrm{Na}]^{+}:$277.0841, found: 277.0846 . Mp: $81-82{ }^{\circ} \mathrm{C}$.

((2R,3S)-3-(4-Isopropylphenyl)oxiran-2-yl)-(phenyl)methanone 7d. ${ }^{14 a}$ A white powder; yield $95 \%$, ee $63 \%$; $[\alpha]_{\mathrm{D}}^{25}-88^{\circ}(c 0.3$ in acetone). ${ }^{1} \mathrm{H}$ NMR (400 MHz, $\left.\mathrm{CDCl}_{3}\right) \delta 8.00(\mathrm{~m}, 2 \mathrm{H}, \mathrm{Ar}-\mathrm{H}), 7.60$ (m, 1H, Ar-H), 7.47 (m, 2H, Ar-H), $7.28(\mathrm{~m}, 4 \mathrm{H}, \mathrm{Ar}-\mathrm{H}), 4.31$ (d, J= $1.9 \mathrm{~Hz}, 1 \mathrm{H}, \mathrm{CH}), 4.05$ (d, J = $1.8 \mathrm{~Hz}, 1 \mathrm{H}, \mathrm{CH}), 2.93(\mathrm{~m}, 1 \mathrm{H}, \mathrm{CH})$, $1.26\left(\mathrm{~d}, J=6.9 \mathrm{~Hz}, 6 \mathrm{H}, \mathrm{CH}_{3}\right)$. HPLC: OD-H column, 90\% hexanes, $10 \%{ }^{\mathrm{i}} \mathrm{PrOH}, 1.0 \mathrm{~mL} \min ^{-1}, t_{\mathrm{r}}$ (minor) $=5.7 \mathrm{~min}, t_{\mathrm{r}}$ (major) $=$ 6.4 min. HRMS (ESI, $m / z$ ) calcd for $\mathrm{C}_{18} \mathrm{H}_{18} \mathrm{O}_{2} \mathrm{Na}[\mathrm{M}+\mathrm{Na}]^{+}$: 289.1204, found: 289.1205 . Mp: $74-76{ }^{\circ} \mathrm{C}$.

((2R,3S)-3-(2-Methoxyphenyl)oxiran-2-yl)-(phenyl)methanone 7e. ${ }^{14 a}$ A white powder; yield $97 \%$, ee $83 \%$; $[\alpha]_{\mathrm{D}}^{25}-94^{\circ}(c 0.3$ in acetone). ${ }^{1} \mathrm{H}$ NMR $\left(400 \mathrm{MHz}, \mathrm{CDCl}_{3}\right) \delta 8.05(\mathrm{~m}, 2 \mathrm{H}, \mathrm{Ar}-\mathrm{H}), 7.62(\mathrm{t}$, $J=7.4 \mathrm{~Hz}, 1 \mathrm{H}, \mathrm{Ar}-\mathrm{H}), 7.49(\mathrm{t}, J=7.7 \mathrm{~Hz}, 2 \mathrm{H}, \mathrm{Ar}-\mathrm{H}), 7.31(\mathrm{~m}, 2 \mathrm{H}$, $\mathrm{Ar}-\mathrm{H}), 7.00$ (t, $J=7.5 \mathrm{~Hz}, 1 \mathrm{H}, \mathrm{Ar}-\mathrm{H}), 6.92$ (d, $J=8.2 \mathrm{~Hz}, 1 \mathrm{H}, \mathrm{Ar}-\mathrm{H})$, $4.39(\mathrm{~d}, J=1.8 \mathrm{~Hz}, 1 \mathrm{H}, \mathrm{CH}), 4.20$ (d, $J=1.9 \mathrm{~Hz}, 1 \mathrm{H}, \mathrm{CH}), 3.83(\mathrm{~s}$, $3 \mathrm{H}, \mathrm{CH}_{3}$ ). HPLC: OD-H column, 90\% hexanes, $10 \%{ }^{\mathrm{i}} \mathrm{PrOH}, 1.0$ $\mathrm{mL} \min ^{-1}, 8.3 \mathrm{~min}$ (minor), 10.1 (major). $t_{\mathrm{r}}$ (minor) $=8.3 \mathrm{~min}, t_{\mathrm{r}}$ (major) $=10.1 \mathrm{~min}$. HRMS (ESI, $m / z$ ) calcd for $\mathrm{C}_{16} \mathrm{H}_{14} \mathrm{O}_{3} \mathrm{Na}[\mathrm{M}+$ $\mathrm{Na}]^{+}:$277.0841, found: 277.08443 . Mp: $89-90{ }^{\circ} \mathrm{C}$.

((2R,3S)-3-(2-Methphenyl)oxiran-2-yl)-(phenyl)methanone $7 \mathbf{f}^{\mathbf{1 4 a}}$ A white powder; yield $96 \%$, ee $73 \%$; $[\alpha]_{\mathrm{D}}^{25}-77^{\circ}\left(c 0.3\right.$ in acetone). ${ }^{1} \mathrm{H}$ NMR (400 MHz, $\left.\mathrm{CDCl}_{3}\right) \delta 8.05(\mathrm{~m}, 2 \mathrm{H}, \mathrm{Ar}-\mathrm{H}), 7.63(\mathrm{~m}, 1 \mathrm{H}, \mathrm{Ar}-\mathrm{H})$, $7.50(\mathrm{t}, J=7.7 \mathrm{~Hz}, 2 \mathrm{H}, \mathrm{Ar}-\mathrm{H}), 7.34$ (dd, $J=6.8,2.3 \mathrm{~Hz}, 1 \mathrm{H}, \mathrm{Ar}-\mathrm{H})$, 7.26 (m, 2H, Ar-H), $7.19(\mathrm{~m}, 1 \mathrm{H}, \mathrm{Ar}-\mathrm{H}), 4.21(\mathrm{t}, J=1.8 \mathrm{~Hz}, 2 \mathrm{H}, \mathrm{CH})$, $2.36\left(\mathrm{~s}, 3 \mathrm{H}, \mathrm{CH}_{3}\right)$. HPLC: OD-H column, $90 \%$ hexanes, $10 \%{ }^{\mathrm{i}} \mathrm{PrOH}$, $1.0 \mathrm{~mL} \min ^{-1}, t_{\mathrm{r}}$ (minor) $=6.8 \mathrm{~min}, t_{\mathrm{r}}$ (major) $=7.7 \mathrm{~min}$. HRMS (ESI, $m / z$ ) calcd for $\mathrm{C}_{16} \mathrm{H}_{14} \mathrm{O}_{2} \mathrm{Na}[\mathrm{M}+\mathrm{Na}]^{+}: 261.2758$, found: 261.2759. Mp: $70-72{ }^{\circ} \mathrm{C}$.

(4-Methylphenyl)-[(2R,3S)-3-(4-isopropylphenyl)-2-oxiran]methanone $7 g .{ }^{14 a}$ A white powder; yield 95\%, ee 57\%; $[\alpha]_{\mathrm{D}}^{25}-97^{\circ}\left(c 0.3\right.$ in acetone). ${ }^{1} \mathrm{H} \mathrm{NMR}\left(400 \mathrm{MHz}, \mathrm{CDCl}_{3}\right) \delta 7.83$ $(\mathrm{d}, J=8.2 \mathrm{~Hz}, 2 \mathrm{H}, \mathrm{Ar}-\mathrm{H}), 7.20(\mathrm{~m}, 6 \mathrm{H}, \mathrm{Ar}-\mathrm{H}), 4.20(\mathrm{~d}, J=1.9 \mathrm{~Hz}$, $1 \mathrm{H}, \mathrm{CH}), 3.95(\mathrm{~d}, J=1.8 \mathrm{~Hz}, 1 \mathrm{H}, \mathrm{CH}), 2.84(\mathrm{~m}, 1 \mathrm{H}, \mathrm{CH}), 2.33$ (s, $\left.3 \mathrm{H}, \mathrm{CH}_{3}\right), 1.18\left(\mathrm{~d}, J=6.9 \mathrm{~Hz}, 6 \mathrm{H}, \mathrm{CH}_{3}\right)$. HPLC: OD-H column,

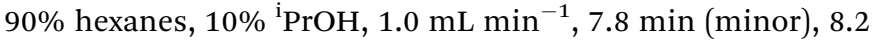
(major). $t_{\mathrm{r}}(\operatorname{minor})=7.8 \mathrm{~min}, t_{\mathrm{r}}$ (major) $=8.2 \mathrm{~min}$. HRMS (ESI, 
$m / z$ ) calcd for $\mathrm{C}_{19} \mathrm{H}_{20} \mathrm{O}_{2} \mathrm{Na}[\mathrm{M}+\mathrm{Na}]^{+}$: 303.1361, found: 303.1367. Mp: $64-67{ }^{\circ} \mathrm{C}$.

(3-Methoxyphenyl)-[(2R,3S)-3-phenyloxiran-2-yl]-methanone 7h. ${ }^{14 a}$ A white powder; yield $93 \%$, ee $37 \%$; $[\alpha]_{\mathrm{D}}^{25}-49^{\circ}(c 0.3$ in acetone). ${ }^{1} \mathrm{H}$ NMR $\left(400 \mathrm{MHz}, \mathrm{CDCl}_{3}\right) \delta 7.57(\mathrm{dd}, J=4.9,3.6 \mathrm{~Hz}$, $1 \mathrm{H}, \mathrm{Ar}-\mathrm{H}), 7.52(\mathrm{~m}, 1 \mathrm{H}, \mathrm{Ar}-\mathrm{H}), 7.38(\mathrm{~m}, 6 \mathrm{H}, \mathrm{Ar}-\mathrm{H}), 7.16(\mathrm{~m}, 1 \mathrm{H}$, $\mathrm{Ar}-\mathrm{H}), 4.29$ (d, $J=1.9 \mathrm{~Hz}, 1 \mathrm{H}, \mathrm{CH}), 4.07$ (d, $J=1.8 \mathrm{~Hz}, 1 \mathrm{H}, \mathrm{CH})$, $3.84\left(\mathrm{~s}, 3 \mathrm{H}, \mathrm{CH}_{3}\right)$. HPLC: OD-H column, $90 \%$ hexanes, $10 \%$

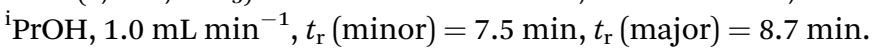
HRMS (ESI, $m / z$ ) calcd for $\mathrm{C}_{16} \mathrm{H}_{14} \mathrm{O}_{3} \mathrm{Na}[\mathrm{M}+\mathrm{Na}]^{+}$: 277.0841, found: 277.0849 . Mp: $74-76{ }^{\circ} \mathrm{C}$.

((2R,3S)-3-(4-Fluorophenyl)oxiran-2-yl)-(phenyl)methanone $7 \mathbf{i}^{14 a}$ A white powder; yield $93 \%$, ee $77 \%$; $[\alpha]_{\mathrm{D}}^{25}-98^{\circ}(c 0.3$ in acetone). ${ }^{1} \mathrm{H}$ NMR $\left(400 \mathrm{MHz}, \mathrm{CDCl}_{3}\right) \delta 7.93(\mathrm{~m}, 2 \mathrm{H}, \mathrm{Ar}-\mathrm{H}), 7.56$ $(\mathrm{m}, 1 \mathrm{H}, \mathrm{Ar}-\mathrm{H}), 7.44(\mathrm{~m}, 4 \mathrm{H}, \mathrm{Ar}-\mathrm{H}), 7.17$ (dd, $J=6.2,4.5 \mathrm{~Hz}, 2 \mathrm{H}$, $\mathrm{Ar}-\mathrm{H}), 4.17$ (d, $J=1.8 \mathrm{~Hz}, 1 \mathrm{H}, \mathrm{CH}), 3.98(\mathrm{~d}, J=1.7 \mathrm{~Hz}, 1 \mathrm{H}, \mathrm{CH})$. HPLC: OD-H column, $90 \%$ hexanes, $10 \%{ }^{\mathrm{i}} \mathrm{PrOH}, 1.0 \mathrm{~mL} \mathrm{~min}^{-1}$, $t_{\mathrm{r}}($ minor $)=8.4 \mathrm{~min}, t_{\mathrm{r}}($ major $)=9.4 \mathrm{~min}$. HRMS $(\mathrm{ESI}, \mathrm{m} / \mathrm{z})$ calcd for $\mathrm{C}_{15} \mathrm{H}_{11} \mathrm{FO}_{2} \mathrm{Na}[\mathrm{M}+\mathrm{Na}]^{+}$: 265.0641, found: 265.0647. Mp: 91.5-94.2 ${ }^{\circ} \mathrm{C}$.

((2R,3S)-3-(4-Chlorophenyl)oxiran-2-yl)-(phenyl)methanone $7 \mathbf{j}^{14 a}$ A white powder; yield $95 \%$, ee $71 \%$; $[\alpha]_{\mathrm{D}}^{25}-80^{\circ}(c 0.3$ in acetone). ${ }^{1} \mathrm{H}$ NMR (400 MHz, $\left.\mathrm{CDCl}_{3}\right) \delta 8.01(\mathrm{~m}, 2 \mathrm{H}, \mathrm{Ar}-\mathrm{H}), 7.63$ (m, 1H, Ar-H), 7.49 (m, 2H, Ar-H), $7.35(\mathrm{~m}, 2 \mathrm{H}, \mathrm{Ar}-\mathrm{H}), 7.10(\mathrm{~m}$, $2 \mathrm{H}, \mathrm{Ar}-\mathrm{H}), 4.26$ (s, 1H, CH), 4.07 (d, $J=1.8 \mathrm{~Hz}, 1 \mathrm{H}, \mathrm{CH})$. HPLC: OD-H column, $90 \%$ hexanes, $10 \%{ }^{\mathrm{i}} \mathrm{PrOH}, 1.0 \mathrm{~mL} \mathrm{~min}{ }^{-1}, t_{\mathrm{r}}$ $($ minor $)=8.0 \mathrm{~min}, t_{\mathrm{r}}($ major $)=8.8 \mathrm{~min}$. HRMS $(\mathrm{ESI}, \mathrm{m} / \mathrm{z})$ calcd for $\mathrm{C}_{15} \mathrm{H}_{11} \mathrm{ClO}_{2} \mathrm{Na}$ [M $\left.+\mathrm{Na}\right]^{+}: 281.0345$, found: 281.0349. Mp: 114 ${ }^{\circ} \mathrm{C}$.

((2R,3S)-3-(4-Bromophenyl)oxiran-2-yl)-(phenyl)methanone $7 \mathbf{k}^{14 a}$ A white powder; yield $92 \%$, ee $67 \% ;[\alpha]_{\mathrm{D}}^{25}-78^{\circ}(c 0.3$ in acetone). ${ }^{1} \mathrm{H}$ NMR (400 MHz, $\left.\mathrm{CDCl}_{3}\right) \delta 8.00(\mathrm{~m}, 2 \mathrm{H}, \mathrm{Ar}-\mathrm{H}), 7.63$ $(\mathrm{m}, 1 \mathrm{H}, \mathrm{Ar}-\mathrm{H}), 7.49$ (dd, $J=10.7,4.8 \mathrm{~Hz}, 2 \mathrm{H}, \mathrm{Ar}-\mathrm{H}), 7.38(\mathrm{~m}, 2 \mathrm{H}$, $\mathrm{Ar}-\mathrm{H}), 7.31(\mathrm{~m}, 2 \mathrm{H}, \mathrm{Ar}-\mathrm{H}), 4.25(\mathrm{~d}, J=1.8 \mathrm{~Hz}, 1 \mathrm{H}, \mathrm{CH}), 4.06(\mathrm{~d}, J$ $=1.8 \mathrm{~Hz}, 1 \mathrm{H}, \mathrm{CH})$. HPLC: OD-H column, $90 \%$ hexanes, $10 \%$ ${ }^{\mathrm{i}} \mathrm{PrOH}, 1.0 \mathrm{~mL} \mathrm{~min}{ }^{-1}, t_{\mathrm{r}}($ minor $)=7.7 \mathrm{~min}, t_{\mathrm{r}}($ major $)=8.2 \mathrm{~min}$. HRMS (ESI, $m / z$ ) calcd for $\mathrm{C}_{15} \mathrm{H}_{11} \mathrm{BrO}_{2} \mathrm{Na}[\mathrm{M}+\mathrm{Na}]^{+}: 324.9840$, found: 324.9843 . Mp: $89-90{ }^{\circ} \mathrm{C}$.

((2R,3S)-3-(3-Chlorophenyl)oxiran-2-yl)-(phenyl)methanone 71. ${ }^{14 a}$ A white powder; yield 98\%, ee $61 \% ;[\alpha]_{\mathrm{D}}^{25}-78^{\circ}(c 0.3$ in acetone). ${ }^{1} \mathrm{H}$ NMR $\left(400 \mathrm{MHz}, \mathrm{CDCl}_{3}\right) \delta 8.00(\mathrm{~m}, 2 \mathrm{H}, \mathrm{Ar}-\mathrm{H}), 7.63$ $(\mathrm{m}, 1 \mathrm{H}, \mathrm{Ar}-\mathrm{H}), 7.49(\mathrm{~m}, 2 \mathrm{H}, \mathrm{Ar}-\mathrm{H}), 7.38(\mathrm{~m}, 2 \mathrm{H}, \mathrm{Ar}-\mathrm{H}), 7.30(\mathrm{~m}$, $2 \mathrm{H}, \mathrm{Ar}-\mathrm{H}), 4.25(\mathrm{~d}, J=1.9 \mathrm{~Hz}, 1 \mathrm{H}, \mathrm{CH}), 4.06(\mathrm{~d}, J=1.8 \mathrm{~Hz}, 1 \mathrm{H}$, CH). HPLC: OD-H column, $90 \%$ hexanes, $10 \%{ }^{\mathrm{i}} \mathrm{PrOH}, 1.0$ $\mathrm{mL} \min ^{-1}, t_{\mathrm{r}}($ minor $)=8.0 \mathrm{~min}, t_{\mathrm{r}}$ (major) $=8.8 \mathrm{~min}$. HRMS (ESI, $m / z$ ) calcd for $\mathrm{C}_{15} \mathrm{H}_{11} \mathrm{ClO}_{2} \mathrm{Na}[\mathrm{M}+\mathrm{Na}]^{+}: 281.0345$, found: 281.0346. Mp: $74-75{ }^{\circ} \mathrm{C}$.

(3-Chlorophenyl)-[(2R,3S)-3-phenyloxiran-2-yl]-methanone $7 \mathbf{m}^{14 a}$ A white powder; yield $97 \%$, ee $61 \%$; $[\alpha]_{\mathrm{D}}^{25}-89^{\circ}(c 0.3$ in acetone). ${ }^{1} \mathrm{H}$ NMR $\left(400 \mathrm{MHz}, \mathrm{CDCl}_{3}\right) \delta 8.03(\mathrm{~m}, 2 \mathrm{H}, \mathrm{Ar}-\mathrm{H}), 7.48$ (m, 2H, Ar-H), 7.38 (m, 5H, Ar-H), $4.23(\mathrm{~d}, J=1.9 \mathrm{~Hz}, 1 \mathrm{H}, \mathrm{CH})$, $4.08(\mathrm{~d}, J=1.8 \mathrm{~Hz}, 1 \mathrm{H}, \mathrm{CH})$. HPLC: OD-H column, $97 \%$ hexanes, $3 \%{ }^{\mathrm{i}} \mathrm{PrOH}, 1.0 \mathrm{~mL} \mathrm{~min}{ }^{-1}, t_{\mathrm{r}}($ minor $)=21.1 \mathrm{~min}, t_{\mathrm{r}}$ (major) $=$ 22.0 min. HRMS (ESI, $m / z)$ calcd for $\mathrm{C}_{15} \mathrm{H}_{11} \mathrm{ClO}_{2} \mathrm{Na}[\mathrm{M}+\mathrm{Na}]^{+}$: 281.0345, found: 281.0354 . Mp: $67-68^{\circ} \mathrm{C}$.

[(2R,3S)-3-Phenyloxiran-2-yl] (thien-2-yl)-methanone $7 \mathrm{n} .{ }^{14 a} \mathrm{~A}$ white powder; yield $99 \%$, ee $65 \%$; $[\alpha]_{\mathrm{D}}^{25}-43^{\circ}$ (c0.3 in acetone).
${ }^{1} \mathrm{H}$ NMR $\left(400 \mathrm{MHz}, \mathrm{CDCl}_{3}\right) \delta 7.97(\mathrm{dd}, J=3.9,1.0 \mathrm{~Hz}, 1 \mathrm{H}, \mathrm{CH})$, $7.72(\mathrm{dd}, J=4.9,1.0 \mathrm{~Hz}, 1 \mathrm{H}, \mathrm{CH}), 7.34(\mathrm{~m}, 5 \mathrm{H}, \mathrm{Ar}-\mathrm{H}), 7.15$ (dd, $J$ $=4.9,3.9 \mathrm{~Hz}, 1 \mathrm{H}, \mathrm{CH}), 4.15(\mathrm{~d}, J=1.7 \mathrm{~Hz}, 1 \mathrm{H}, \mathrm{CH}), 4.08(\mathrm{~d}, J=$ $1.8 \mathrm{~Hz}, 1 \mathrm{H}, \mathrm{CH})$. HPLC: OD-H column, $90 \%$ hexanes, $10 \%$ ${ }^{\mathrm{i}} \mathrm{PrOH}, 1.0 \mathrm{~mL} \mathrm{~min}{ }^{-1}, t_{\mathrm{r}}($ minor $)=10.8 \mathrm{~min}, t_{\mathrm{r}}$ (major) $=$ 10.1 min. HRMS (ESI, $\mathrm{m} / \mathrm{z}$ ) calcd for $\mathrm{C}_{13} \mathrm{H}_{10} \mathrm{O}_{2} \mathrm{SNa}[\mathrm{M}+\mathrm{Na}]^{+}$: 253.0229, found: 253.0233 . Mp: $54-56^{\circ} \mathrm{C}$.

[(2R,3S)-3-Phenyloxiran-2-yl](furan-2-yl)-methanone $70^{15 a} \mathrm{~A}$ white powder; yield $95 \%$, ee $71 \%$; $[\alpha]_{\mathrm{D}}^{25}-57^{\circ}$ ( $c 0.3$ in acetone). ${ }^{1} \mathrm{H} \mathrm{NMR}\left(400 \mathrm{MHz}, \mathrm{CDCl}_{3}\right) \delta 7.66(\mathrm{~d}, J=1.0 \mathrm{~Hz}, 1 \mathrm{H}, \mathrm{CH}), 7.45(\mathrm{~d}$, $J=3.6 \mathrm{~Hz}, 1 \mathrm{H}, \mathrm{CH}), 7.35(\mathrm{~m}, 5 \mathrm{H}, \mathrm{Ar}-\mathrm{H}), 6.59(\mathrm{dd}, J=3.6,1.7 \mathrm{~Hz}$, $1 \mathrm{H}, \mathrm{CH}), 4.14(\mathrm{~s}, 2 \mathrm{H}, \mathrm{CH})$. HPLC: OD-H column, $90 \%$ hexanes, $10 \%{ }^{\mathrm{i}} \mathrm{PrOH}, 1.0 \mathrm{~mL} \mathrm{~min}{ }^{-1}, t_{\mathrm{r}}(\operatorname{minor})=10.8 \mathrm{~min}, t_{\mathrm{r}}$ (major) $=$ 11.3 min. HRMS (ESI, $m / z$ ) calcd for $\mathrm{C}_{13} \mathrm{H}_{10} \mathrm{O}_{3} \mathrm{Na}[\mathrm{M}+\mathrm{Na}]^{+}$: 237.0528, found: 237.0533 . Mp: $44-47^{\circ} \mathrm{C}$.

(2R,3'S)-3'-Phenyl-3,4-dihydro-1H-spiro[naphthalene-2, $2^{\prime}$ oxiran]-1-one $7 \mathbf{p}^{14 b}$ A white powder; yield $43 \%$, ee $83 \%$; $[\alpha]_{\mathrm{D}}^{25}-96^{\circ}$ (c0.3 in acetone). ${ }^{1} \mathrm{H}$ NMR $\left(400 \mathrm{MHz}, \mathrm{CDCl}_{3}\right) \delta 8.10$ (dd, $J=7.8,0.9 \mathrm{~Hz}, 1 \mathrm{H}, \mathrm{Ar}-\mathrm{H}), 7.51(\mathrm{~m}, 1 \mathrm{H}, \mathrm{Ar}-\mathrm{H}), 7.37(\mathrm{~m}, 6 \mathrm{H}$, $\mathrm{Ar}-\mathrm{H}), 7.22(\mathrm{~d}, J=7.6 \mathrm{~Hz}, 1 \mathrm{H}, \mathrm{Ar}-\mathrm{H}), 4.35$ (s, 1H, CH), $2.82(\mathrm{dd}, J$ $\left.=8.5,4.1 \mathrm{~Hz}, 2 \mathrm{H}, \mathrm{CH}_{2}\right), 2.43\left(\mathrm{~m}, 1 \mathrm{H}, \mathrm{CH}_{2}\right), 1.85\left(\mathrm{~m}, 1 \mathrm{H}, \mathrm{CH}_{2}\right)$. HPLC: OD-H column, $97 \%$ hexanes, $3 \%{ }^{\mathrm{i}} \mathrm{PrOH}, 1.0 \mathrm{~mL} \mathrm{~min}{ }^{-1}, t_{\mathrm{r}}$ $($ minor $)=19.5 \mathrm{~min}, t_{\mathrm{r}}($ major$)=21.1 \mathrm{~min}$. HRMS (ESI, $\left.\mathrm{m} / z\right)$ calcd for $\mathrm{C}_{17} \mathrm{H}_{14} \mathrm{O}_{2} \mathrm{Na}[\mathrm{M}+\mathrm{Na}]^{+}:$273.0891, found: 273.0897. Mp: $73.5-75.5{ }^{\circ} \mathrm{C}$.

(2R,3'S)-3'-(4-Methoxyphenyl)-3,4-dihydro-1H-spiro[naphthalene-2,2'-oxiran]-1-one $\mathbf{7 q} \mathbf{q}^{14 b} \mathrm{~A}$ white powder; yield $43 \%$, ee $87 \% ;[\alpha]_{\mathrm{D}}^{25}-111^{\circ}\left(c 0.3\right.$ in acetone). ${ }^{1} \mathrm{H}$ NMR $\left(400 \mathrm{MHz}, \mathrm{CDCl}_{3}\right)$ $\delta 8.10(\mathrm{~d}, J=7.8 \mathrm{~Hz}, 1 \mathrm{H}, \mathrm{Ar}-\mathrm{H}), 7.51(\mathrm{~m}, 1 \mathrm{H}, \mathrm{Ar}-\mathrm{H}), 7.35(\mathrm{t}, J=$ $7.5 \mathrm{~Hz}, 1 \mathrm{H}, \mathrm{Ar}-\mathrm{H}), 7.29$ (d, $J=8.6 \mathrm{~Hz}, 2 \mathrm{H}, \mathrm{Ar}-\mathrm{H}), 7.22(\mathrm{~d}, J=$ $7.6 \mathrm{~Hz}, 1 \mathrm{H}, \mathrm{Ar}-\mathrm{H}), 6.93(\mathrm{dd}, J=6.6,4.8 \mathrm{~Hz}, 2 \mathrm{H}, \mathrm{Ar}-\mathrm{H}), 4.30(\mathrm{~s}$, $1 \mathrm{H}, \mathrm{CH}), 3.83\left(\mathrm{~s}, 3 \mathrm{H}, \mathrm{CH}_{3}\right), 2.83\left(\mathrm{dd}, J=8.5,4.0 \mathrm{~Hz}, 2 \mathrm{H}, \mathrm{CH}_{2}\right.$ ), $2.44\left(\mathrm{~m}, 1 \mathrm{H}, \mathrm{CH}_{2}\right), 1.86\left(\mathrm{~m}, 1 \mathrm{H}, \mathrm{CH}_{2}\right)$. HPLC: OD-H column, $97 \%$

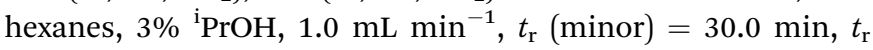
(major) $=28.7 \mathrm{~min}$. HRMS (ESI, $m / z$ ) calcd for $\mathrm{C}_{18} \mathrm{H}_{16} \mathrm{O}_{3} \mathrm{Na}[\mathrm{M}+$ $\mathrm{Na}]^{+}:$303.0997, found: 303.0999 . Mp: $76-79^{\circ} \mathrm{C}$.

(2R,3'S)-3'-(4-Methphenyl)-3,4-dihydro-1 $H$-spiro[naphthalene-2,2'-oxiran]-1-one $7 \mathbf{r}^{14 b} \mathrm{~A}$ white powder; yield $41 \%$, ee $81 \%$; $[\alpha]_{\mathrm{D}}^{25}-41^{\circ}$ (c0.3 in acetone). ${ }^{1} \mathrm{H}$ NMR $\left(400 \mathrm{MHz}, \mathrm{CDCl}_{3}\right) \delta 8.11$ (dd, $J=7.9,1.0 \mathrm{~Hz}, 1 \mathrm{H}, \mathrm{Ar}-\mathrm{H}), 7.51(\mathrm{~m}, 1 \mathrm{H}, \mathrm{Ar}-\mathrm{H}), 7.36(\mathrm{t}, J=$ $7.5 \mathrm{~Hz}, 1 \mathrm{H}, \mathrm{Ar}-\mathrm{H}), 7.25(\mathrm{~m}, 6 \mathrm{H}, \mathrm{Ar}-\mathrm{H}), 4.32(\mathrm{~s}, 1 \mathrm{H}, \mathrm{CH}), 2.83(\mathrm{dd}$, $\left.J=8.5,4.1 \mathrm{~Hz}, 2 \mathrm{H}, \mathrm{CH}_{2}\right), 2.44\left(\mathrm{~m}, 1 \mathrm{H}, \mathrm{CH}_{2}\right), 2.38\left(\mathrm{~s}, 3 \mathrm{H}, \mathrm{CH}_{3}\right)$, $1.86\left(\mathrm{~m}, 1 \mathrm{H}, \mathrm{CH}_{2}\right)$. HPLC: OD-H column, $90 \%$ hexanes, $10 \%$ ${ }^{\mathrm{i}} \mathrm{PrOH}, 1.0 \mathrm{~mL} \mathrm{~min}{ }^{-1}, t_{\mathrm{r}}($ minor $)=14.9 \mathrm{~min}, t_{\mathrm{r}}$ (major) $=$ 20.9 min. HRMS (ESI, $m / z$ ) calcd for $\mathrm{C}_{18} \mathrm{H}_{16} \mathrm{O}_{2} \mathrm{Na}[\mathrm{M}+\mathrm{Na}]^{+}$: 287.1048, found: 287.1051 . Mp: $165-169^{\circ} \mathrm{C}$.

(2R,3'S)-3'-(3-Methphenyl)-3,4-dihydro-1H-spiro[naphthalene-2, $\mathbf{2}^{\prime}$-oxiran]-1-one $\mathbf{7 s .}{ }^{17} \mathrm{~A}$ white powder; yield $99 \%$, ee $80 \%$; $[\alpha]_{\mathrm{D}}^{25}-78^{\circ}\left(c 0.3\right.$ in acetone). $1 \mathrm{H}$ NMR $\left(400 \mathrm{MHz}, \mathrm{CDCl}_{3}\right) \delta 8.11$ (m, 1H, Ar-H), $7.51(\mathrm{~m}, 1 \mathrm{H}, \mathrm{Ar}-\mathrm{H}), 7.36(\mathrm{t}, J=7.6 \mathrm{~Hz}, 1 \mathrm{H}, \mathrm{Ar}-\mathrm{H})$, $7.25(\mathrm{~m}, 2 \mathrm{H}, \mathrm{Ar}-\mathrm{H}), 7.17(\mathrm{~m}, 3 \mathrm{H}, \mathrm{Ar}-\mathrm{H}), 4.32(\mathrm{~s}, 1 \mathrm{H}, \mathrm{CH}), 2.83$ (dd, $\left.J=8.4,4.1 \mathrm{~Hz}, 2 \mathrm{H}, \mathrm{CH}_{2}\right), 2.44\left(\mathrm{~m}, 1 \mathrm{H}, \mathrm{CH}_{2}\right), 2.37(\mathrm{~s}, 3 \mathrm{H}$, $\left.\mathrm{CH}_{3}\right), 1.87 \mathrm{~m}, 1 \mathrm{H}, \mathrm{CH}_{2}$ ). HPLC: OD-H column, 90\% hexanes, $10 \%{ }^{\mathrm{i}} \mathrm{PrOH}, 1.0 \mathrm{~mL} \mathrm{~min}{ }^{-1}, t_{\mathrm{r}}($ minor $)=7.8 \mathrm{~min}, t_{\mathrm{r}}$ (major) $=$ 6.9 min. HRMS (ESI, $m / z$ ) calcd for $\mathrm{C}_{18} \mathrm{H}_{16} \mathrm{O}_{2} \mathrm{Na}[\mathrm{M}+\mathrm{Na}]^{+}$: 287.1048, found: 287.1052 . Mp: $96-98{ }^{\circ} \mathrm{C}$. 
(2R,3'S)-3'-(2-Methphenyl)-3,4-dihydro-1H-spiro[naphthalene-2, $\mathbf{2}^{\prime}$-oxiran]-1-one $7 \mathbf{t t}^{17} \mathrm{~A}$ white powder; yield 99\%, ee $87 \%$; $[\alpha]_{\mathrm{D}}^{25}-88^{\circ}$ (c0.3 in acetone). ${ }^{1} \mathrm{H}$ NMR $\left(400 \mathrm{MHz}, \mathrm{CDCl}_{3}\right) \delta 8.13$ $(\mathrm{dd}, J=7.9,1.0 \mathrm{~Hz}, 1 \mathrm{H}, \mathrm{Ar}-\mathrm{H}), 7.53(\mathrm{~m}, 1 \mathrm{H}, \mathrm{Ar}-\mathrm{H}), 7.36(\mathrm{dd}, J=$ 12.4, $5.4 \mathrm{~Hz}, 2 \mathrm{H}, \mathrm{Ar}-\mathrm{H}$ ), 7.25 (m, 3H, Ar-H), 7.18 (dd, $J=6.4$, $2.2 \mathrm{~Hz}, 1 \mathrm{H}, \mathrm{Ar}-\mathrm{H}), 4.36(\mathrm{~s}, 1 \mathrm{H}, \mathrm{CH}), 2.83\left(\mathrm{~m}, 2 \mathrm{H}, \mathrm{CH}_{2}\right), 2.37(\mathrm{~m}$, $\left.1 \mathrm{H}, \mathrm{CH}_{2}\right), 2.25\left(\mathrm{~s}, 3 \mathrm{H}, \mathrm{CH}_{3}\right), 1.74\left(\mathrm{~m}, 1 \mathrm{H}, \mathrm{CH}_{2}\right)$. HPLC: OD-H column, $90 \%$ hexanes, $10 \%{ }^{\mathrm{i}} \mathrm{PrOH}, 1.0 \mathrm{~mL} \mathrm{~min}^{-1}, t_{\mathrm{r}}$ (minor) $=6.6 \mathrm{~min}, t_{\mathrm{r}}$ (major) $=6.9 \mathrm{~min}$. HRMS (ESI, $\mathrm{m} / z$ ) calcd for $\mathrm{C}_{18} \mathrm{H}_{16} \mathrm{O}_{2} \mathrm{Na}[\mathrm{M}+\mathrm{Na}]^{+}:$287.1048, found: 287.1051 . Mp: 114$116^{\circ} \mathrm{C}$.

$\left(2 R, 3^{\prime} S\right)-3^{\prime}$-(4-Fluorophenyl)-3,4-dihydro-1H-spiro[naphthalene-2, $2^{\prime}$-oxiran]-1-one $7 \mathbf{u}^{17}$ A yellow powder; yield 38\%, ee $81 \%$; $[\alpha]_{\mathrm{D}}^{25}-54^{\circ}\left(c 0.3\right.$ in acetone). ${ }^{1} \mathrm{H}$ NMR $\left(400 \mathrm{MHz}, \mathrm{CDCl}_{3}\right) \delta 8.10(\mathrm{~d}$, $J=7.9 \mathrm{~Hz}, 1 \mathrm{H}, \mathrm{Ar}-\mathrm{H}), 7.53(\mathrm{t}, J=7.5 \mathrm{~Hz}, 1 \mathrm{H}, \mathrm{Ar}-\mathrm{H}), 7.36(\mathrm{~m}, 3 \mathrm{H}$, $\mathrm{Ar}-\mathrm{H}), 7.23$ (d, $J=7.7 \mathrm{~Hz}, 1 \mathrm{H}, \mathrm{Ar}-\mathrm{H}), 7.09$ (t, $J=8.4 \mathrm{~Hz}, 2 \mathrm{H}, \mathrm{Ar}-$ $\mathrm{H}), 4.34(\mathrm{~s}, 1 \mathrm{H}, \mathrm{CH}), 2.85\left(\mathrm{~m}, 2 \mathrm{H}, \mathrm{CH}_{2}\right), 2.43\left(\mathrm{~m}, 1 \mathrm{H}, \mathrm{CH}_{2}\right), 1.82$ (m, $1 \mathrm{H}, \mathrm{CH}_{2}$ ). HPLC: OD-H column, $90 \%$ hexanes, $10 \%{ }^{\mathrm{i}} \mathrm{PrOH}$, $1.0 \mathrm{~mL} \mathrm{~min}^{-1}, t_{\mathrm{r}}$ (minor) $=8.6 \mathrm{~min}, t_{\mathrm{r}}$ (major) $=8.1 \mathrm{~min}$. HRMS (ESI, $m / z$ ) calcd for $\mathrm{C}_{17} \mathrm{H}_{13} \mathrm{FO}_{2} \mathrm{Na}[\mathrm{M}+\mathrm{Na}]^{+}: 291.0907$, found: 291.0913. Mp: $99.5-101.5{ }^{\circ} \mathrm{C}$.

$\left(2 S, 3^{\prime} R\right)-3^{\prime}$-(4-Chlorophenyl)-3,4-dihydro-1H-spiro[naphthalene-2, $2^{\prime}$-oxiran]-1-one $7 \mathbf{v} .{ }^{14 b}$ A white powder; yield 32\%, ee 83\%; $[\alpha]_{\mathrm{D}}^{25}-102^{\circ}$ (c0.3 in acetone). ${ }^{1} \mathrm{H} \mathrm{NMR}\left(400 \mathrm{MHz}, \mathrm{CDCl}_{3}\right) \delta 8.10$ (dd, $J=7.9,1.0 \mathrm{~Hz}, 1 \mathrm{H}, \mathrm{Ar}-\mathrm{H}), 7.53(\mathrm{~m}, 1 \mathrm{H}, \mathrm{Ar}-\mathrm{H}), 7.34(\mathrm{~m}, 5 \mathrm{H}$, $\mathrm{Ar}-\mathrm{H}), 7.24$ (d, J=7.6 Hz, 1H, Ar-H), 4.33 (s, 1H, CH), 2.83 (m, $\left.2 \mathrm{H}, \mathrm{CH}_{2}\right), 2.44\left(\mathrm{~m}, 1 \mathrm{H}, \mathrm{CH}_{2}\right), 1.81\left(\mathrm{~m}, 1 \mathrm{H}, \mathrm{CH}_{2}\right)$.HPLC: OD-H column, 90\% hexanes, $10 \% \mathrm{iPrOH}, 1.0 \mathrm{~mL} \mathrm{~min}^{-1}, t_{\mathrm{r}}$ (minor) $=9.1 \mathrm{~min}, t_{\mathrm{r}}$ (major) $=8.5 \mathrm{~min}$. HRMS (ESI, $\mathrm{m} / z$ ) calcd for $\mathrm{C}_{17} \mathrm{H}_{13} \mathrm{ClO}_{2} \mathrm{Na}[\mathrm{M}+\mathrm{Na}]^{+}: 307.0502$, found: 307.0509. Mp: 138$142{ }^{\circ} \mathrm{C}$.

$\left(2 R, 3^{\prime} S\right)-3^{\prime}$-(4-Bromophenyl)-3,4-dihydro-1H-spiro[naphthalene-2, $\mathbf{2}^{\prime}$-oxiran]-1-one $7 \mathbf{w} .^{\mathbf{1 4 b}}$ A white powder; yield $49 \%$, ee $80 \% ;[\alpha]_{\mathrm{D}}^{25}-113^{\circ}$ (c0.3 in acetone). ${ }^{1} \mathrm{H}$ NMR (400 $\mathrm{MHz}, \mathrm{CDCl}_{3}$ ) $\delta 8.10(\mathrm{~d}, J=7.8 \mathrm{~Hz}, 1 \mathrm{H}, \mathrm{Ar}-\mathrm{H}), 7.53(\mathrm{dd}, J=6.6,4.4 \mathrm{~Hz}, 3 \mathrm{H}, \mathrm{Ar}-$ $\mathrm{H}), 7.37(\mathrm{t}, J=7.5 \mathrm{~Hz}, 1 \mathrm{H}, \mathrm{Ar}-\mathrm{H}), 7.25(\mathrm{~m}, 3 \mathrm{H}, \mathrm{Ar}-\mathrm{H}), 4.31(\mathrm{~s}, 1 \mathrm{H}$, $\mathrm{CH}), 2.83\left(\mathrm{~m}, 2 \mathrm{H}, \mathrm{CH}_{2}\right), 2.44\left(\mathrm{~m}, 1 \mathrm{H}, \mathrm{CH}_{2}\right), 1.81\left(\mathrm{~m}, 1 \mathrm{H}, \mathrm{CH}_{2}\right)$. HPLC: OD-H column, 90\% hexanes, $10 \%{ }^{\mathrm{i}} \mathrm{PrOH}, 1.0 \mathrm{~mL} \mathrm{~min}{ }^{-1}$, $t_{\mathrm{r}}($ minor $)=9.5 \mathrm{~min}, t_{\mathrm{r}}$ (major) $=8.9 \mathrm{~min}$. HRMS (ESI, $\left.m / z\right)$ calcd for $\mathrm{C}_{17} \mathrm{H}_{13} \mathrm{BrO}_{2} \mathrm{Na}[\mathrm{M}+\mathrm{Na}]^{+}$: 350.9997, found: 351.0002. Mp: 122-125 ${ }^{\circ} \mathrm{C}$.

$\left(2 R, 3^{\prime} S\right)-3^{\prime}$-(3-Chlorophenyl)-3,4-dihydro-1H-spiro[naphthalene-2, $2^{\prime}$-oxiran]-1-one $7 x{ }^{17} \mathrm{~A}$ white powder; yield 99\%, ee $80 \%$; $[\alpha]_{\mathrm{D}}^{25}-99^{\circ}$ (c0.3 in acetone). ${ }^{1} \mathrm{H}$ NMR (400 $\left.\mathrm{MHz}, \mathrm{CDCl}_{3}\right) \delta 8.10$ (dd, $J=7.9,1.1 \mathrm{~Hz}, 1 \mathrm{H}, \mathrm{Ar}-\mathrm{H}), 7.53(\mathrm{~m}, 1 \mathrm{H}, \mathrm{Ar}-\mathrm{H}), 7.35(\mathrm{~m}, 4 \mathrm{H}$, $\mathrm{Ar}-\mathrm{H}), 7.26$ (m, 2H, Ar-H), $4.34(\mathrm{~s}, 1 \mathrm{H}, \mathrm{CH}), 2.35\left(\mathrm{~m}, 2 \mathrm{H}, \mathrm{CH}_{2}\right)$, $2.44\left(\mathrm{~m}, 1 \mathrm{H}, \mathrm{CH}_{2}\right), 1.84\left(\mathrm{~m}, 1 \mathrm{H}, \mathrm{CH}_{2}\right)$. HPLC: OD-H column, 90\%

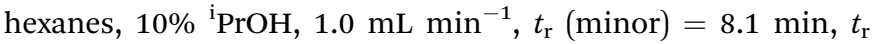
(major) $=7.6 \mathrm{~min}$. HRMS (ESI, $m / z)$ calcd for $\mathrm{C}_{17} \mathrm{H}_{13} \mathrm{ClO}_{2} \mathrm{Na}[\mathrm{M}+$ $\mathrm{Na}]^{+}: 307.0502$, found: 307.0506. Mp: $94-97^{\circ} \mathrm{C}$.

$\left(2 R, 3^{\prime} S\right)-3^{\prime}$-(2-Chlorophenyl)-3,4-dihydro-1H-spiro[naphthalene-2, $\mathbf{2}^{\prime}$-oxiran]-1-one $7 \mathbf{y} .{ }^{17} \mathrm{~A}$ white powder; yield 99\%, ee $80 \%$; $[\alpha]_{\mathrm{D}}^{25}-110^{\circ}$ (c0.3 in acetone). ${ }^{1} \mathrm{H}$ NMR $\left(400 \mathrm{MHz}, \mathrm{CDCl}_{3}\right) \delta 8.14$ (dd, $J=7.9,1.2 \mathrm{~Hz}, 1 \mathrm{H}, \mathrm{Ar}-\mathrm{H}), 7.53(\mathrm{~m}, 1 \mathrm{H}, \mathrm{Ar}-\mathrm{H}), 7.44(\mathrm{~m}, 1 \mathrm{H}$, Ar-H), 7.39 (m, 2Hm, Ar-H), 7.34 (m, 2H, Ar-H), 7.25 (d, $J=$ $7.7 \mathrm{~Hz}, 1 \mathrm{H}, \mathrm{Ar}=\mathrm{H}), 4.44(\mathrm{~s}, 1 \mathrm{H}, \mathrm{CH}), 3.02\left(\mathrm{~m}, 1 \mathrm{H}, \mathrm{CH}_{2}\right), 2.85(\mathrm{~m}$,
1H, $\left.\mathrm{CH}_{2}\right), 2.39\left(\mathrm{~m}, 1 \mathrm{H}, \mathrm{CH}_{2}\right), 1.64\left(\mathrm{~m}, 1 \mathrm{H}, \mathrm{CH}_{2}\right)$. HPLC: OD-H column, 97\% hexanes, $3 \%{ }^{\mathrm{i}} \mathrm{PrOH}, 1.0 \mathrm{~mL} \mathrm{~min}^{-1}, t_{\mathrm{r}}$ (minor) $=$ $21.1 \mathrm{~min}, t_{\mathrm{r}}$ (major) $=19.5 \mathrm{~min}$. HRMS (ESI, $\mathrm{m} / \mathrm{z}$ ) calcd for $\mathrm{C}_{17} \mathrm{H}_{13} \mathrm{ClO}_{2} \mathrm{Na}[\mathrm{M}+\mathrm{Na}]^{+}: 307.0502$, found: 307.0506 . Mp: $132-$ $134{ }^{\circ} \mathrm{C}$.

\section{Conflicts of interest}

The authors declare no competing financial interest.

\section{Acknowledgements}

We gratefully acknowledge financial support from the National Natural Science Foundation of China (Grant No. 21572151), the project of Scientific and Technologic Infrastructure of Suzhou (SZS201708), and PAPD.

\section{Notes and references}

1 P. G. Cozzi, Chem. Soc. Rev., 2004, 33, 410-421.

2 (a) M. Kitamura, S. Suga, K. Kawai and R. Noyori, J. Am. Chem. Soc., 1986, 108, 6071-6072; (b) R. Noyori and M. Kitamura, Angew. Chem., Int. Ed. Engl., 1991, 30, 49-69; (c) M. Quirmbach, A. Kless, J. Holz, B. Tararov and A. Börner, Tetrahedron: Asymmetry, 1999, 10, 1803-1811; (d) A. Kless, R. Kadyrov, A. Börner, J. Holz and H. B. Kagan, Tetrahedron Lett., 1995, 36, 4601-4602; (e) E. F. Dimauro and M. C. Kozlowski, Org. Lett., 2001, 3(19), 3053-3056.

3 (a) E. F. DiMauro and M. C. Kozlowski, J. Am. Chem. Soc., 2002, 124, 12668-12670; (b) C. Garcfa, L. K. LaRochelle and P. J. Walsh, J. Am. Chem. Soc., 2002, 124, 10970-10971; (c) Y. N. Belokon, B. Green, N. S. Ikonnikov, M. North and V. I. Tararov, Tetrahedron Lett., 1999, 40, 8147-8149; (d) K. Yabu, S. Masumoto, M. Kanai, D. P. Curran and M. Shibasaki, Tetrahedron Lett., 2002, 43, 2923-2925; (e) S. Masumoto, M. Suzuki, M. Kanai and M. Shibasaki, Tetrahedron Lett., 2002, 43, 8647-8650; $(f)$ S. K. Tian and L. Deng, J. Am. Chem. Soc., 2001, 123, 6195-6197; $(g)$ H. Deng, M. P. Snapper and A. H. Hoveyda, Angew. Chem., Int. Ed., 2002, 41, 1009-1011; (h) F. X. Chen, X. M. Feng, B. Qin, G. L. Zhang and Y. Z. Jiang, Org. Lett., 2003, 5(6), 949-952.

4 (a) D. A. Evans, C. S. Burgey, N. A. Paras, T. Vojkovsky and S. W. Tregay, J. Am. Chem. Soc., 1998, 120, 5824-5825; (b) D. A. Evans, S. W. Tregay, C. S. Burgey, N. A. Paras and T. Vojkovsky, J. Am. Chem. Soc., 2000, 122, 7936-7943; (c) Y. Yuan, X. Zhang and K. Ding, Angew. Chem., Int. Ed., 2003, 42, 5478-5480; (d) D. A. Evans and J. Wu, J. Am. Chem. Soc., 2005, 127, 8006-8007; (e) G. E. Huston, A. H. Dave and V. H. Rawal, Org. Lett., 2007, 9, 3869-3872.

5 (a) F. D. Denes, A. Perez-Luna and F. Chemla, Chem. Rev., 2010, 110, 2366-2368; (b) B. K. Corkey and F. D. Toste, J. Am. Chem. Soc., 2005, 125, 17168-17169; (c) T. Yang, A. Ferrali, F. Sladojevich, L. Campbell and D. Dixon, J. Am. Chem. Soc., 2009, 131, 9140-9141; (d) A. Matsuzawa, T. Mashiko, N. Kumagai and M. Shibasaki, Angew. Chem., 
Int. Ed., 2011, 50, 7616-7618; (e) S. Shaw and J. D. White, J. Am. Chem. Soc., 2014, 136, 13578-13581.

6 (a) B. Gong, Q. Meng, T. Su, M. Lian, Q. Wang and Z. Gao, Synlett, 2009, 2009, 2659-2661; (b) Y. Cai, M. Lian, Z. Li and Q. Meng, Tetrahedron, 2012, 68, 7973-7978; (c) Z. Li, M. Lian, F. Yang, Q. Meng and Z. Gao, Eur. J. Org. Chem., 2014, 2014, 3491-3495; (d) M. Lian, Z. Li, J. Du, Q. Meng and Z. Gao, Eur. J. Org. Chem., 2010, 2010, 6525-6529; (e) F. Yang, J. N. Zhao, X. F. Tang, G. L. Zhou, W. Z. Song and Q. W. Meng, Org. Lett., 2017, 19, 448-451.

7 (a) R. I. Kureshy, N. H. Khan, S. H. R. Abdi, S. T. Patel and R. V. Jasra, Tetrahedron: Asymmetry, 2001, 12, 433-437; (b) R. I. Kureshy, N. H. Khan, S. H. R. Abdi, I. Ahmed, S. Singh and R. V. Jasra, J. Mol. Catal. A: Chem., 2003, 219, 1-7; (c) R. I. Kureshy, N. H. Khan, S. H. R. Abdi, S. Singh, I. Ahmad, R. V. Jasra and A. P. Vyas, J. Catal., 2004, 224, 229-235.

8 S. Biswajit, L. Mei-Huey and T. V. RajanBabu, J. Org. Chem., 2007, 72, 8648-8655.

9 P. N. O'Shaughnessy, D. K. Paul, M. Colin, M. G. Kevin and S. Peter, Chem. Commun., 2003, 1770-1771.

10 S. Handa, V. Gnanadesikan, S. Matsunaga and M. Shibasaki, J. Am. Chem. Soc., 2007, 129, 4900-4901.

11 (a) M. Bougauchi, S. Watanabe, T. Arai, H. Sasai and M. Shibasaki, J. Am. Chem. Soc., 1997, 119, 2329-2330; (b) T. Nemoto, T. Ohshima and M. Shibasaki, J. Am. Chem. Soc., 2001, 123, 2725-2727; (c) T. Ohshima, T. Nemoto,
S.-y. Tosaki, H. Kakei, V. Gnanadesikan and M. Shibasaki, Tetrahedron, 2003, 59, 10485-10488; (d) S. Matsunaga, T. Kinoshita, S. Okada, S. Harada and M. Shibasaki, J. Am. Chem. Soc., 2004, 126, 7559-7561; (e) H. Kakei, R. Tsuji, T. Ohshima and M. Shibasaki, J. Am. Chem. Soc., 2005, 127, 8962-8963.

12 (a) K. Daikai, T. Hayano, R. Kino, H. Furuno, T. Kagawa and J. Inanaga, Chirality, 2003, 15, 83-88; (b) X.-W. Wang, L. Shi, M.-X. Li and K.-L. Ding, Angew. Chem., Int. Ed., 2005, 44, 6362-6366.

13 Y. Y. Chu, X. H. Liu, W. Li, X. L. Hu, L. L. Lin and X. M. Feng, Chem. Sci., 2012, 3, 1996-1998.

14 (a) Q. Qian, Y. Tan, B. Zhao, T. Feng, Q. Shen and Y. Yao, Org. Lett., 2014, 16, 4516-4519; (b) C. Zeng, D. Yuan, B. Zhao and Y. Yao, Org. Lett., 2015, 17, 2242-2245.

15 (a) L. Canali and D. C. Sherrington, Chem. Soc. Rev., 1999, 28, 85-89; (b) R. Breinbauer and E. N. Jacobsen, Angew. Chem., Int. Ed., 2000, 39, 3604-3607; (c) E. M. McMarrigle and D. G. Gilheany, Chem. Rev., 2005, 105, 1563-1566.

16 (a) O. Cussó, I. Garcia-Bosch, X. Ribas, J. LIoret-Fillot and M. Costas, J. Am. Chem. Soc., 2013, 135, 14871-14878; (b) R. V. Ottenbacher, D. G. Samsonenko, E. P. Talsi and K. P. Bryliakov, ACS Catal., 2014, 1599-1606; (c) D. Shen, B. Qin, D. Xu, C. Xia and W. Sun, Org. Lett., 2016, 18, 372375.

17 W. Bin, W. Shoufang, X. Chungu and S. Wei, Chem.-Eur. J., 2012, 18, 7332-7335. 